\title{
An overview of coupled cluster theory and its applications in physics
}

\author{
R. F. Bishop \\ Department of Mathematics, University of Manchester Institute of Science and Technology, P.O. \\ Box 88, Manchester M60 1QD, UK
}

Received December 15, 1990; received in revised form March 4, 1991/Accepted March 19, 1991

Summary. What has since become known as the normal coupled cluster method (NCCM) was invented about thirty years ago to calculate ground-state energies of closed-shell atomic nuclei. Coupled cluster (CC) techniques have since been developed to calculate excited states, energies of open-shell systems, density matrices and hence other properties, sum rules, and the sub-sum-rules that follow from imbedding linear response theory within the NCCM. Further extensions deal both with systems at nonzero temperature ar.1 with general dynamical behaviour. More recently, a new version of $\mathrm{CC}$ theory, the so-called extended coupled cluster method (ECCM) has been introduced. It has the potential to describe such global phenomena as phase transitions, spontaneous symmetry breaking, states of topological excitation, and nonequilibrium behaviour. CC techniques are now widely recognized as providing one of the most universally applicable, most powerful, and most accurate of all microscopic $a b$ initio methods in quantum many-body theory. The number of successful applications within physics is now impressively large. In most such cases the numerical results are either the best or among the best available. A typical case is the electron gas, where the $\mathrm{CC}$ results for the correlation energy agree over the entire metallic density range to within less than 1 millihartree (or $<1 \%$ ) with the essentially exact Green's function Monte Carlo results. The role of CC theory within modern quantum many-body theory is first surveyed, by a comparison with other techniques. Its full range of applications in physics is then reviewed. These include problems in nuclear physics, both for finite nuclei and infinite nuclear matter; the electron gas; various integrable and nonintegrable models; various relativistic quantum field theories; and quantum spin chain and lattice models. Particular applications of the ECCM include the quantum hydrodynamics of a zero-temperature, strongly-interacting condensed Bose fluid; a charged impurity in a polarizable medium (e.g., positron annihilation in metals); and various anharmonic oscillator and spin systems.

Key words: Coupled cluster theory - Normal coupled cluster method (NCCM) - Extended coupled cluster method (ECCM) - Quantum many-body techniques - Electron gas - Atomic nuclei - Nuclear matter-Quantum field theory Quantum hydrodynamics - Positron annihilation 


\section{Introduction}

Coupled cluster theory is nowadays widely recognized by the quantum chemistry community as being one of the most powerful and most accurate microscopic formulations of the quantum many-electron problem that underpins their discipline at the fundamental level. Precise descriptions of atoms and molecules require a very accurate incorporation of the most important effects of the electronic correlations, many of which are often subtly intertwined with each other at the quantitative level necessary before $a b$ initio microscopic methods can attain their ultimate aim of being predictive. As an overall benchmark goal in this regard, quantum chemists aim to calculate correlation energies, for example, to an accuracy of one millihartree $(1 \mathrm{mH})$.

As several other articles in this issue attest, and as various other recent reviews and collections of articles (see, e.g., Refs. [1,2]) detail at some length, the coupled cluster method (CCM) is now at both the formal and computational stages of development where these goals are being met for ever more complex polyatomic molecules, and for a wide range of properties and phenomena of chemical interest. The CCM is therefore increasingly becoming the method of first choice for a variety of problems connected with molecular structure and molecular spectra. Examples include the calculation of ionization potentials, electron affinities, ground-state expectation values of operators other than the Hamiltonian, excitation energies, and energy gradients (for use, for example, in searching potential energy surfaces to predict vibrational spectra and to locate transition states in decomposition reactions).

Although the CCM was invented over thirty years ago by Coester and Kümmel $[3,4]$, it was not until nearly ten years later that it was introduced into quantum chemistry by Čižek [5]. It was then less than twenty years ago that the first $a b$ initio comparison was made between the corresponding coupled cluster (CC) and configuration-interaction (CI) wavefunctions of a specific molecular system, namely the $\mathrm{BH}_{3}$ molecule using a simple minimal basis set [6]. It is interesting now to recall that this particular model was chosen since it was known that triexcited clusters made a large contribution to the energy. In fact, these authors demonstrated that the most important such contributions also came from the real (connected) triple excitations which could not be decomposed into (disconnected) single or double excitation components. The development of the computational technology that has now recently allowed an accurate evaluation of these triple excitations, still had to wait another fifteen years $[7,8]$.

The simplest implementation of the CCM in quantum chemistry is the coupled cluster doubles (CCD) approximation which includes all biexcited clusters with respect to some antisymmetric Slater determinant (single-reference) state. The explicit CCD equations were first written down by Čizek [5]. General purpose suites of $a b$ initio CCD programmes for molecules were developed by two groups $[9,10]$ in 1978 , and became widely available thereafter. Only in the case when the reference state is an antisymmetrized product of so-called maximum overlap orbitals do the monoexcited clusters identically vanish. Otherwise their incorporation along with the biexcited clusters is achieved by the so-called coupled cluster singles and doubles (CCSD) approximation, the first implementation of which was reported in 1982 [11]. As we have already seen, Noga and Bartlett [7] have only recently reported the first full coupled cluster singles, doubles and triples (CCSDT) results. By comparing with the corresponding full 
CI results they have now demonstrated that this method is the most accurate (single-reference) method currently available in quantum chemistry.

Furthermore, it is nowadays widely accepted that any further increases in accuracy within quantum chemistry are also likely to arise from advances within the CCM itself. As we have seen, accurate and efficient computational implementations of the CCM for atoms and molecules have been available for only about the last ten years. (By comparison, the CI method has been an effective computational tool for over twice that period, although even here its essential multireference and unitary group extensions have only existed for about ten years.) Finally, the extensions from the original $\mathrm{CC}$ single-reference approach for ground-state energies to deal, inter alia, with energy gradients, excited states, and multireference methods are all areas of intense current development in quantum chemistry, as other articles in this volume will attest, and to which the interested reader may turn for further references to the quantum chemistry literature.

From the above description it should be apparent that $\mathrm{CC}$ technology is likely to continue to play an important role in $a b$ initio computational quantum chemistry. Hence it seems particularly appropriate to remind ourselves that the $\mathrm{CCM}$ has by now also received very wide attention outside chemistry. In view of the many parallel developments and applications of the method that have been made in physics, for both finite and extended systems, which may therefore also lend themselves for use in chemical applications, it seems expecially apposite to review these other developments. Indeed, while quantum chemists are undoubtedly the biggest users of the CCM techniques, and while they have been responsible for several essential theoretical contributions and most of the powerful computational advances which have turned the formalism into the viable tool it now is, it is also true that much of the progress and many of the formal developments of the CCM have been made elsewhere.

The intention of the present article is therefore to review in very broad outline the large panoply of formal developments that have taken place under the umbrella title of CC theory, and to survey the associated spectrum of applications that have been made within the purview of physics, as opposed to chemistry. Other articles in the present volume and other recent reviews (see, e.g., Refs. [2, 12]) undertake the same aim from within chemistry, and so I will not discuss further any application to atoms, molecules, and other closely related models or areas of interest. Even so, the field of CC theory and its applications has by now grown so large that it is not possible to attempt a complete survey in an article of this size. It must therefore be clearly stressed from the outset that this review is intended to be indicative and illustrative rather than exhaustive. In many ways it may be viewed as a more detailed companion and follow-up article to an earlier introductory article on the same theme by the present author and one of the co-inventors of the CCM [13]. In that article we set the stage on which the present review is based.

The remainder of the present paper is organized as follows. The particular niche that the CCM currently occupies within modern quantum many-body theory is first outlined in Sect. 2. In order to appreciate its central significance we briefly review there the main other microscopic general-purpose tools that are available to the many-body theorist, so that comparisons can be made. In Sect. 3 we then attempt to provide an overview of all of the main formal strands that when woven together have become what is nowadays referred to collectively as $\mathrm{CC}$ theory or the CCM.

It is thereafter convenient to discuss the specific applications of the CCM within physics according to various broad areas of specialization. Although there 
is clearly no unique way of making these subdivisions, we find it useful to discuss in turn, in Sects. 4-8 respectively, the main applications that have been made to nuclear physics; the one-component Coulomb plasmas (e.g., the electron gas or "jellium") of special interest within (metallic) solid-state physics; various model many-body problems of interest within mathematical physics; quantum field theory; and quantum spin chain and lattice models. Various other more formal applications of $\mathrm{CC}$ techniques to such problems as the quantum fluid mechanics of a strongly interacting (zero-temperature) condensed Bose fluid, and a charged impurity in a polarizable medium (of relevance, for example, to the important experimental tool of positron annihilation in metals and other forms of condensed matter), are also briefly sketched in Sect. 9. Finally, we conclude in Sect. 10 with some remarks concerning possible future directions.

\section{Role of the CCM in modern quantum many-body theory}

Many-body physics or quantum many-body theory is not the rather specialized subfield of physics that it is sometimes believed to be. On the contrary, at least at the most fundamental or microscopic level in each of the energy ranges which are traditionally used to delimit and characterize particular branches of physics, we are nearly always faced with many-particle situations. Thus, it is clear that in fields like molecular, solid-state, and nuclear physics most of the fundamental objects of discourse are interacting many-body systems. But even in elementary particle physics one is usually dealing with more than one particle. For example, at some level of reality a nucleon is made up of three quarks interacting via gluons and surrounded by a cloud of mesons, which are themselves composed of quark-antiquark pairs. Even more fundamentally, even the "physical vacuum" of any quantum field theory is endowed with an enormously complex infinite many-body structure due to the virtual excitation of particles.

Thus, it is quite clear that although techniques to deal with interacting many-body systems are likely to have separately arisen from specific physical contexts, they may also be of more general interest. The CCM and its birth within nuclear physics is only one example. Nevertheless, if we limit ourselves to comparisons with other fundamental and "universal" tools, the number of competing methods is surprisingly small. Before we enumerate them, however, we emphasize that there exist also many other macroscopic or phenomenological or approximate or empirical descriptions and techniques, which have proven successful in one or other context. Some of these have also been essential for the later development of more fundamental techniques. They are, on the other hand, excluded from our present considerations because, by their very nature, they are "specialist" tools which are tailor-made for a particular system or application, and because our emphasis here is on techniques that have a more universal appeal.

Clearly those methods which are intrinsically exact and which therefore lend themselves to systematic sequences of approximations, have a particular intellectual appeal. Indeed one could justifiably argue that this feature is both a prerequisite and a hallmark of any truly microscopic $a b$ initio formulation of the general many-body problem. Occupying a special place between theory and experiment are several Monte Carlo methods (e.g., the Green's function, diffusion, and path-integral varieties) that solve the $N$-body Schrödinger equation directly by stochastic simulation, typically for $N \lesssim 300$ (and see, e.g., Refs. 
[14-17]). In those cases and for those properties where they can be applied, these methods provide a set of unique benchmarks against which other ab initio calculations may be measured, since these results may be considered to be exact to within well-defined statistical errors (together with an extrapolation error associated with taking the bulk limit, $N \rightarrow \infty$, for extensive systems).

The remaining microscopic techniques fall into the six main classes: (i) time-independent perturbation theory (TIPT); (ii) Green's function (GF) or propagator methods; (iii) variational methods; (iv) the correlated basis function (CBF) method; (v) the configuration-interaction (CI) and other closely related "generalized shell-model" methods; and (vi) the coupled cluster method (CCM). The first two of these methods are perturbative in origin. The TIPT technique initially provides an expansion in powers of some relevant coupling constant associated with the interaction term in the Hamiltonian. Individual terms in the resulting series may be considered graphically in terms of the usual Goldstone diagrams. Various infinite partial resummations are often performed out of necessity, either because the series diverges badly or is otherwise ill-defined from the outset. Well-known examples include the ladder-diagram summation (for hard-core or other strongly-repulsive short-range potentials) which leads to the Bethe-Brueckner-Goldstone $G$-matrix expansion or the hole-line expansion (and see, e.g., Refs [18-20]); and the bubble- or ring-diagram summation of the random phase approximation (RPA) [21, 22] (for long-range potentials of the Coulomb type).

Even in such rearranged or partially resummed examples, one is still ultimately forced in practice to neglect all terms that are presumed to be unimportant or that cannot easily be handled. Furthermore, it is usually difficult in realistic applications to justify the retention of certain terms at the expense of neglecting others. The history of microscopic nuclear theory, for example, is littered with incorrect or misleading calculations which amply illustrate the danger of the blind inclusion of extra diagrams, as has been pointed out many times by Kümmel and his coworkers $[23,24]$. In quantum chemistry, the TIPT techniques provide the usual means of doing many-body perturbation theory (MBPT) or what in chemistry has increasingly become known as Møller-Plesset perturbation theory. In chemical applications it is usual in MBPT to include all terms through some given finite order in the electron interactions. Full fourthorder, MBPT(4), calculations are now commonplace [25].

By comparison with TIPT methods, GF techniques are rooted in time-dependent perturbation theory, and hence rely again on expansions in powers of the coupling constant. They may also be formulated as time-dependent equations for matrix elements which describe the propagation of groups of particles within the many-body system. These (multi-particle) Green's functions or propagators, and their perturbative expansions, find a diagrammatic representation in terms of Feynman diagrams. Although both time-dependent and time-independent versions of perturbation theory lead formally to identical (exact) results, at given respective levels of truncation they will certainly differ from each other.

Such propagator methods have been and still are widely used in quantum chemistry (and see, e.g., Refs. [26, 27]), particularly for molecular spectroscopic properties. Nevertheless, on the wider front they suffer from many of the same drawbacks as the TIPT techniques discussed above. Thus, with one possible caveat, neither TIPT nor GF methods are nowadays generally considered within quantum many-body theory to be sufficiently accurate or sufficiently versatile to be candidates for the position of a universal, high-precision method. The only 
real exception to this viewpoint is provided by the so-called parquet diagram, or planar theory, approach within the broader GF scheme.

The ideas behind parquet theory were introduced [28] at around the same time that Coester first introduced the CCM. The specific context and impetus for their introduction was certain problems in particle physics. Similar equations were later used to study X-ray absorption and emission in metals [29]. However, it was not until 1979 that the potential of parquet theory for the study of strongly interacting condensed Bose systems was noted by Ripka [30]. He particularly called attention to the fact that the hypernetted chain (HNC) equations of the Jastrow variational approach, which we discuss in more detail below and which was at that time meeting with considerable success in dealing with such extended bosonic systems as liquid ${ }^{4} \mathrm{He}$, shared severable very desirable features with the parquet equations. He remarked that just as the HNC equations of variational theory treated simultaneously and consistently both the longand short-range correlations, so too would the parquet equations in perturbation theory, if only they could be solved.

This astute observation of Ripka provided the stimulus for the 1982 study of Jackson, Lande and Smith [31], which examined the parquet equations for bosons in great detail. They also showed how these otherwise rather formidable equations could be rendered tractable, and hence solved numerically, by the introduction of simple localizing approximations.

The basic concepts of parquet theory are both simple and appealing. The method first focusses on the effective two-body interaction, which it then expresses in terms of a large and physically interesting class of Feynman diagrams. These so-called parquet diagrams are a particular self-consistent sum of ring, ladder, and vertex correction terms for the two-body Green's function. Most important physically is the fact that the two-body (i.e., particle-particle and hole-hole) ladder diagrams and the particle-hole ring diagrams are iterated together in a maximal fashion. (Indeed in this one important respect parquet theory has a conceptual and practical advantage over the CCM which in its lowest CCD approximation also includes among many others all ring and all ladder diagrams, together with some but not all of their joint iterates.) Although the full two-body parquet equations are highly complex, their correspondingly approximated local counterparts were applied to such model bosonic systems as liquid ${ }^{4} \mathrm{He}$ using the Lennard-Jones potential, and neutron matter treated as a Bose system interacting via the Reid soft-core ${ }^{1} S_{0}$ interaction. Not surprisingly in view of Ripka's comments, good agreement was found with the corresponding optimized Jastrow $\mathrm{HNC}$ results.

At the formal level it was later demonstrated [32] that a similar, but nonetheless distinct, local form of the full two-body parquet equations is identical to optimized Jastrow HNC theory. This result was particularly important since it provided a bridge between the otherwise very disjoint perturbative approaches of TIPT and GF theory on the one hand, and variational approaches on the other. Later formal developments have included a possible extension to fermionic systems [33, 34]; the inclusion of three-body terms [35]; and parquet perturbation theory [36] for bosons, as an expansion in the difference between the exact and approximate propagators, in order to improve systematically upon the local parquet equations. Interesting connections between parquet theory and Baym-Kadanoff theory [37] have also been made [38]. This latter approach is noteworthy in that it uses an initial approximation for the two-body vertex to construct from it a conserving vertex, namely one which conserves particle number, momentum, and energy. 
Despite what is now a fairly large corpus of formal developments, the parquet method has not yet been widely applied and tested. Nevertheless, it clearly deserves to be, and it must be considered as a potential candidate for the position of a universal tool in quantum many-body theory. We have stressed it here for just these reasons.

We turn next to the use of variational methods in quantum many-body theory. Perhaps the simplest of these approaches are based on a trial wavefunction of the (Bijl-Dingle-) Jastrow type [39]. The early calculations of this sort relied on various cluster expansions of the ensuing approximate matrix elements [39-41]. Later on it was realized that the variational approaches may themselves be formulated diagrammatically $[30,42,43]$. This feature has been of considerable help in constructing such powerful approximations as the Percus-Yevick and HNC summations (which have their origins in the classical theory of liquids) and their variants, both for bosons and fermions [30, 44-46]. Various articles and reviews concerning the use of variational methods in quantum many-body theory exist (and see, e.g., Refs. [30,47-50]). The 1979 article by Clark [47] is particularly recommended as what is perhaps still the standard review of the variational theory of extended nuclear matter. Similar reviews (and see, e.g., Ref. [51]) also exist for applications to few-body systems.

The variational approaches sketched above suffer from two fundamental flaws. In the first place the various partial summations of the graphs considered by such approximation schemes as HNC give methods which lose one of the most attractive features of variational formulations, namely that they yield estimates for the energy which are upper bounds to the exact ground-state energy. Secondly, even a complete summation of graphs (or a variational Monte Carlo evaluation of the corresponding expectation values) for a given trial wavefunction, say of the Jastrow type, gives only the exact variational result and not the true ground state. This second deficiency may be removed in principle by including more general state-dependent correlations and higher-order correlation functions of the Feenberg type [52]. Alternatively, and more generally, one may extend the Jastrow wavefunction to a complete set of correlated basis functions (CBF),

The CBF method was introduced some thirty years ago by Feenberg and his collaborators [44,53], and has since then been largely developed by Clark and his coworkers [54-60]. A very brief qualitative survey of the method has been given recently by the present author [61]. It also contains many more citations of the original CBF literature. The central ingredient of the CBF method is the direct incorporation of the most important interparticle correlations which are believed to characterize the system under consideration, into the approximate wavefunctions on which the microscopic description is based. At its simplest level the method considers only a single configuration and hence reduces to ordinary variational theory. As we have seen above, the latter further simplifies to Jastrow theory if the simplest reasonable choice of correlation operator is made in terms of a symmetric product over all pairs in the system of state-independent two-body correlation functions.

At its most general level, the CBF method constructs for the $N$-body system a multiconfigurational correlated basis $\left\{\left|\Psi_{I}\right\rangle\right\}$ of normalized but generally nonorthogonal state vectors:

$$
\left|\Psi_{I}\right\rangle=\frac{F\left|\Phi_{I}\right\rangle}{\left\langle\Phi_{I}\left|F^{\dagger} F\right| \Phi_{I}\right\rangle^{1 / 2}},
$$


in terms of a correlation operator $F$ applied to a complete orthonormal basis $\left\{\left|\Phi_{I}\right\rangle\right\}$ of model states. The latter usually carry the correct quantum statistics and any essential symmetries of the system. They would hence provide an adequate lowest-order description of the system if it were not strongly interacting. For an application to molecules, for example, the states $\left\{\left|\Phi_{I}\right\rangle\right\}$ would probably be chosen as a set of Slater determinants of some appropriate singleelectron orbitals.

The correlation operator $F$ is thus symmetric in the complete sets of single-particle quantum numbers used to denote the $N$ particles. Most importantly, $F$ also possesses the cluster decomposition property, namely that upon separating one subgroup of particles (say $1,2, \ldots, n$ ) far from the rest $(n+1, n+2, \ldots, N)$ in real space, the operator $F(1,2, \ldots, N)$ decomposes into a product:

$$
F(1, \ldots, N) \rightarrow F^{(n)}(1, \ldots, n) F^{(N-n)}(n+1, \ldots, N) .
$$

It is this property which allows the natural definition of correlation operators $F^{(n)}(1, \ldots, n)$ for $n$-body subsystems with $1 \leqslant n \leqslant N$, in terms of a given $N$-body operator $F \equiv F^{(N)}$. It is also the key ingredient in allowing the derivation of linked cluster expansions for physical quantities.

The simplest choice for $F$ is the state-independent Jastrow form, $F \rightarrow F_{J}$, specified in its usual coordinate-space form as:

$$
F_{J}=\prod_{1 \leqslant i<j \leqslant N} f\left(r_{i j}\right)
$$

where $r_{i j}$ is the relative coordinate for particles $i$ and $j$. The more general Feenberg form, $F \rightarrow F_{F}$, is given in terms of higher-order correlation functions as:

$$
F_{F}=\prod_{i<j} f_{2}\left(r_{i j}\right) \prod_{i<j<k} f_{3}\left(r_{i j}, r_{i k}, r_{j k}\right) \ldots
$$

Whatever choice for $F$ is adopted, the CBF method devolves onto the computation of the matrix elements $H_{I J}$ and $N_{I J}$ of the Hamiltonian $H$ and the unit operator, respectively:

$$
H_{I J}=\left\langle\Phi_{I}\left|F^{\dagger} H F\right| \Phi_{J}\right\rangle ; \quad N_{I J}=\left\langle\Phi_{I}\left|F^{\dagger} F\right| \Phi_{J}\right\rangle .
$$

One assumes that any exact stationary energy eigenstate $|\Xi\rangle, H|\Xi\rangle=E|\Xi\rangle$, may now be (approximately) expanded in the multiconfigurational basis:

$$
|\Xi\rangle=\sum_{J} C_{J}\left|\Psi_{J}\right\rangle
$$

The Schrödinger equation is then decomposed as usual into the coupled set of linear generalized eigenvalue equations:

$$
\sum_{J}\left(H_{I J}-E N_{I J}\right) C_{J}=0,
$$

which have a nontrivial solution for the coefficients $\left\{C_{J}\right\}$ if and only if $E$ satisfies the secular equation:

$$
\operatorname{det}\left(H_{I J}-E N_{I J}\right)=0 .
$$

Clearly, the accurate numerical evaluation of the matrix elements $\left\{H_{I J}\right\}$ and $\left\{N_{I J}\right\}$ is far from trivial. In this context various cluster-expansion techniques 
have been developed [41, 62], which themselves also provided a basis for much of the later diagrammatic analysis of both diagonal elements (expectation values) [42-46] and off-diagonal elements [55]. This latter work further led to the definition of the CBF effective interaction, and to many illuminating connections with conventional diagrammatic MBPT. Exploration of these connections has allowed techniques from standard TIPT as developed for weakly-interacting systems to be taken over into their CBF counterparts for comparable application to strongly-interacting systems (and see, e.g., Ref. [59]).

Finally, however the matrix elements $\left\{H_{I J}\right\}$ and $\left\{N_{I J}\right\}$ are derived, the remaining generalized eigenvalue problem of Eqs. (7) and (8) must then be attacked in a systematic, approximate, or heuristic manner. Various such methods have been developed within the general CBF framework. These include nonorthogonal perturbation theory [54]; straightforward CI-type diagonalization in the space spanned by some subset of the full correlated basis [54,63]; and a correlated version of the usual RPA [21], which now performs a semiclassical treatment of small-amplitude oscillations about an equilibrium correlated configuration $[56,57,59,60]$. There have even been attempts to formulate a generalized version of coupled cluster theory in the CBF basis [64].

We turn then finally to the CI method [65] and the CCM. Both of these remaining microscopic many-body techniques are well known in quantum chemistry, and the latter is our main concern here. For present overview purposes we merely remind the reader that the CI method is basically a straightforward diagonalization of the Hamiltonian in some multiconfigurational subspace of the full many-body Hilbert space. One of its main advantages is that it is also easily and equivalently formulated variationally, in a parametrization that preserves the manifest Hermitian adjoint relationship between corresponding bra and ket wavefunctions. In this way we see that each of the set of approximate energy eigenvalues so obtained provides an upper bound to the corresponding member of the similar set of exact lowest eigenvalues. Furthermore, by the well-known interlacing (or Hylleraas-Undheim) theorem [66], an increase in the size of the multiconfigurational CI subspace is guaranteed to lead to improved estimates, i.e., to ones which are lower (or, in the worst case, unchanged) in energy.

This advantage of the CI method (which is not shared by the CCM) is, however, offset by the disadvantage that the method is not size-extensive [9]. In diagrammatic language, it contains terms which are not linked (connected). For extended systems this deficiency is fatal. In order to guarantee the size-extensivity of the energy of the many-body system, it is clearly necessary that the effective Hamiltonian is separable over the various possible subsystems which arise in the corresponding dissociation limits where they are asymptotically separated to large relative distances so that the interactions between them become vanishingly small. This will certainly be the case if the wave operator is similarly multiplicatively separable. The importance of exactly maintaining the separability of the wavefunction at any subsequent level of approximation has been stressed by Primas [67], although its importance for energy calculations was perhaps first realized by Brueckner [68] when using nondegenerate Rayleigh-Schrödinger MBPT to calculate the ground-state energy of infinite nuclear matter. An obvious method of ensuring that the wave operator is multiplicatively separable is to write it as the exponential of the so-called cluster operator, which in turn is additively separable and hence representable by a sum of connected diagrams. As is by now very well known, this is the central feature of the original CCM as described more fully in Sect. 3. 
From our discussion to date it should be clear that the only existing fully microscopic formulations of quantum many-body theory which are capable of systematic improvement, and which are both widely applicable and capable of high precision (and hence of being "universal" tools), are the CBF method and the CCM. Parquet theory may also fall into this category but it has not yet been sufficiently widely tested to be able to judge. On the other hand, the CBF method has been widely applied to systems as diverse as finite nuclei [56,60,63]; nuclear matter [47,69]; neutron stars [70]; liquid ${ }^{4} \mathrm{He}[69]$; unpolarized $[69,71]$ and polarized [59] liquid ${ }^{3} \mathrm{He}$; the electron gas [71]; various species of electron-spinaligned bulk atomic deuterium [58]; the lattice Hubbard model of strongly interacting electrons [72]; and the $\mathrm{U}(1)_{3}$ lattice gauge model of quantum electrodynamics [73]. The CCM has been at least as widely and at least as successfully and accurately applied in both chemistry and physics. It is our intention in the remainder of this paper to survey the latter applications.

Before doing so, we end the present overview of many-body techniques by noting that the fact that there exists only a small number of fundamental $a b$ initio methods is not something to be regretted. It is widely believed by quantum many-body theorists that much more important nowadays than the development of further techniques is the exploration of the interconnections between existing methods. This is clearly a reflection on the power and universality of the available methods. In this vein we note that there have already been several important confrontations and proposed marriages between existing methods, and we conclude by mentioning some of them.

In the first place, one of the earliest confrontations was provided by the so-called "crisis in nuclear matter theory" which occupied a central place in quantum many-body theory in the 1970 s. Two fundamental issues were involved. Thus, there was a disagreement between the lowest-order Brueckner theory calculations of TIPT and various variational calculations, when both were performed with the same internucleon potential. Furthermore, neither method gave good saturation properties when the best available potentials were used. That crisis has now been essentially resolved (and see, e.g., Ref. [52]) to the mutual advantage of both methods. In particular, the later development of Brueckner theory that was necessary for the resolution has greatly added to our understanding of many-body theory and many-body systems. Another very beneficial outcome of this early confrontation was that the power of the variational and the $C B F$ techniques was thereafter much more widely appreciated. Indeed, the subsequent emphasis placed on the complementary roles played by the variational and the perturbative approaches has also been of importance in many later developments.

Secondly, and continuing in the same spirit, we have already noted the important formal role played by parquet theory [32] in demonstrating the exact equivalence between the optimized HNC approach to the variational Jastrow theory and a particular (approximate) local form of itself. Thirdly, the relationship of the CCM to TIPT as providing at given levels of truncation (e.g., CCD, CCSDT) very sophisticated and intricate resummations of various physically important and infinite classes of Goldstone diagrams, is widely known. A fourth and perhaps less well-known result concerns the relationship of the CI method to the CCM. In particular, the CI method together with the two main modern complete formulations of the CCM which we describe later, and known respectively as the normal (NCCM) and extended (ECCM) versions [74], have been shown [75] to form a natural closed hierarchy of three increasingly 
sophisticated parametrizations in which the underlying amplitudes have increasing degrees of connectedness. In the same spirit, each of the three methods has also been completely reformulated $[74,75]$ via a variational principle.

Fifthly, and at the level of possible proposed marriages between the various methods, we have already mentioned the "correlated coupled cluster theory" [64] which was devised to combine the best features of both the CCM and CBF approaches. It is unfortunate that, to the best of our knowledge, this marriage has not yet been put to the test of a real application. More recently, two other such extensions of the CCM have been discussed. Thus, sixthly, in a recent series of papers $[76,77]$ the CCM has been the subject of intense investigation in order exactly to incorporate translational invariance into it, for use with such light finite systems as the alpha-particle. As a consequence, the emphasis has been to formulate the CCM and the underlying correlations directly in coordinate space rather than in the more usual multiconfigurational Fock-space representation. In this way contact has been made both with more traditional generalized nuclear shell-model calculations of the CI type, with the result that the number of configurations can be dramatically reduced [76], and also with variational approaches [77]. This latter work holds out real possibilities of combining the best elements of the CL, variational and CCM approaches. Finally, and as a seventh example, very recent work [78] has been connected with the incorporation of Jastrow-type correlations into the higher-order cluster terms that are otherwise neglected entirely in the standard (e.g., CCD, CCSDT) CCM truncation schemes.

All of these various partial mergers hold considerable promise for the future. Indeed, it is clear that as both the CBF and CCM techniques in particular become increasingly sophisticated, the differences between them are becoming increasingly blurred.

\section{Overview of $\mathrm{CC}$ theory: the formalism}

The CCM, in its original single-reference formulation [3, 4], was invented as a means of parametrizing the ground ket state of a quantum many-fermion system at zero temperature (and thus in a pure state), and hence of evaluating its energy eigenvalue. It is important to realize, however, that the method has now developed far beyond this level. The range of individual components now contained under the general heading of CCM theory is demonstrated in Fig. 1, in which we have also made a very schematic attempt to indicate the hierarchical structure of the main elements. The reader should be aware that the schema shown is neither unique in its decomposition nor wholly rigorous in the imposition of a logical structure on the resulting components. Nevertheless, we believe that Fig. 1 is useful, at least as an aide memoire in surveying the formal aspects.

Perhaps the most fundamental ingredient for any application of the CCM is the so-called model state $|\Phi\rangle$. Its choice is wide, but it must play the basic role of a cyclic vector, with respect to which we can define two Abelian (i.e., mutually commuting) subalgebras of multiconfigurational creation operators $\left\{C_{I}^{\dagger}\right\}$ and their Hermitian-adjoint destruction operators $\left\{C_{I}\right\}$. Thus, the prime requirement is that arbitrary ket and bra states within the many-body Hilbert space may then be decomposed as the respective linear combinations:

$$
|\Psi\rangle=\sum_{I} \psi_{I} C_{I}^{\dagger}|\Phi\rangle ; \quad\langle\tilde{\Psi}|=\sum_{I} \tilde{\psi}_{I}\langle\Phi| C_{I} .
$$




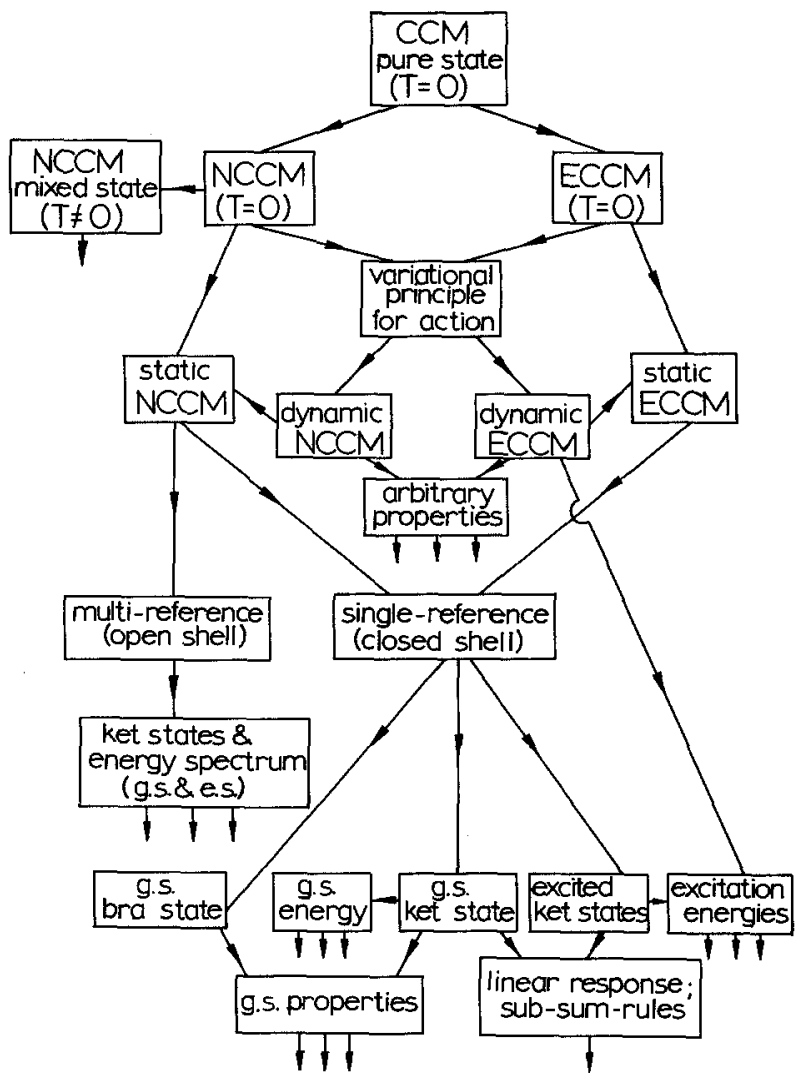

Fig. 1. A schematic overview of the main ingredients of coupled cluster theory, and their hierarchical structure

The set-index $I$ clearly labels a general multiparticle cluster configuration, which in itself defined with respect to the model state $|\Phi\rangle$ in the role of a 'vacuum' or reference state. For the typical number-conserving Fermi system used in quantum chemistry, the standard choice for $|\Phi\rangle$ is usually the single-particle shellmodel (Slater determinant) state formed from an antisymmetrized product of suitably chosen single-particle orbitals. (For an extended translationally invariant system this state becomes the usual filled Fermi sea of plane-wave states.) The creation operators $\left\{C_{l}^{\dagger}\right\}$ then describe configurations formed with respect to this single-reference, noninteracting, closed-shell state by the formation of multiple pairs of single fermions in (particle) orbitals unoccupied in $|\Phi\rangle$ and single vacancies in the corresponding (hole) orbitals occupied in $|\Phi\rangle$.

Nevertheless, this is by no means the only choice. The superconducting Bardeen-Cooper-Schrieffer (BCS) state is an example of a number-nonconserving choice. The accepted wisdom is for $|\Phi\rangle$ to embody the underlying statistics and other symmetry properties of the system, or its particular phase under consideration, so far as is practicable. For extended number-nonconserving bosonic systems the zero-momentum condensate is a standard choice, whereas for field-theoretic systems one might well opt for the bare vacuum in numbernonconserving cases.

Other examples also exist. For example, for such spin-algebraic systems as the Lipkin-Meshkov-Glick (LMG) model [79] which are defined with respect 
to an irreducible SU(2) multiplet of fixed quantum number $j$, and hence spanned by the usual set of $(2 j+1)$ states $\{|j, m\rangle ; m=-j,-j+1, \ldots, j\}$, the state $|\Phi\rangle$ may be chosen as $|\Phi\rangle=|j,-j\rangle$. In this case, the creation operators $\left\{C_{I}^{\dagger}\right\}$ are simply the usual step-up ladder operators, $\left(J_{+}\right)^{m}$, up to suitable multiplicative normalization constants. Similarly, for such spin- $\frac{1}{2}$ magnetic systems as the Heisenberg model defined on a bipartite lattice, $|\Phi\rangle$ may be chosen as the perfectly aligned state (in the ferromagnetic regime) or the Néel state (in the antiferromagnetic regime), for example. For further discussion of these very general algebraic foundations of the CCM the reader is referred to Ref. [80].

The exact ground-state ket wavefunction, $|\Psi\rangle$, is then written in terms of the model state $|\Phi\rangle$ in the standard CCM form as:

$$
|\Psi\rangle=e^{S}|\Phi\rangle
$$

in terms of the cluster correlation operator $S$ (which is more usually denoted as $T$ in quantum chemistry applications). It may itself be decomposed wholly in terms of creation operators as:

$$
S=\sum_{I}^{\prime} s_{I} C_{I}^{\dagger}
$$

where, by definition, the prime on the sum over configurations $\{I\}$ excludes the $I=0$ term corresponding to the identity operator, $C_{0}^{\dagger} \equiv 1$. We thus have the intermediate normalization condition $\langle\Phi \mid \Psi\rangle=\langle\Phi \mid \Phi\rangle=1$. The exact groundstate Schrödinger equation:

$$
H|\Psi\rangle=E|\Psi\rangle,
$$

is then rewritten in the similarity-transformed form:

$$
e^{-S} H e^{S}|\Phi\rangle=E|\Phi\rangle,
$$

which is the hallmark of the CCM. By taking the inner products of Eq. (13) with $|\Phi\rangle$ itself and with the complete set of states $\left\{C_{I}^{\dagger}|\Phi\rangle ; I \neq 0\right\}$ we are thus led respectively to an equation for the ground-state energy eigenvalue $E$ in terms of the cluster correlation coefficients $\left\{s_{I}\right\}$, and a set of formally exact, microscopic, coupled nonlinear equations for these coefficients, in which there appear no macroscopic terms like the energy $E$. These latter equations are all of linked-cluster type, due to the nested commutator expansion:

$$
e^{-S} H e^{S}=H+[H, S]+\frac{1}{2 !}[[H, S], S]+\cdots
$$

and the fact that all of the individual components of $S$ commute with each other, so that each element of $S$ in Eq. (11) is linked directly to the Hamiltonian. Furthermore, the otherwise infinite series of Eq. (14) also always terminates in this case after a finite number of terms, since each term in the second-quantized form of the Hamiltonian contains a finite number of destruction operators (defined with respect to $|\Phi\rangle$ ).

The standard CCM description of excited states has been given, inter alia, by Emrich [81]. Strictly speaking, Eq. (10) describes not only the ground state of Eq. (12), but also any state with the same quantum numbers as the ground state and with nonzero overlap with the model state, $\langle\Phi \mid \Psi\rangle \neq 0$. The possibility of obtaining multiple solutions to the nonlinear ground-state CCM equations has been discussed in some detail for a particular model case in Ref. [82]. For excited states (or more generally, for states with zero overlap with $|\Phi\rangle$ ), we construct the 
respective ket wavefunctions $\left\{\left|\Psi_{\lambda}\right\rangle\right\}$ in the CCM in terms of a set of linear excitation operators $\left\{X^{\lambda}\right\}$ which act on the corresponding exact ground-state ket wavefunction $|\Psi\rangle$ :

$$
\left|\Psi_{\lambda}\right\rangle=X^{\lambda}|\Psi\rangle=X^{\lambda} e^{S}|\Phi\rangle
$$

where $X^{2}$ is again decomposed wholly in terms of creation pieces with respect to $|\Phi\rangle$ :

$$
X^{\lambda}=\sum_{I}^{\prime} x_{I}^{\lambda} C_{I}^{\dagger}
$$

Hence, the operators $X^{\lambda}$ and $S$ commute. The prime on the sum in Eq. (16) ensures that $\left\langle\Phi \mid \Psi_{\lambda}\right\rangle=0$. For extended systems with more than one phase, for example, the so-called ground-state formulation of Eqs. (10)-(11) will generally yield only the lowest state of a given symmetry imposed implicitly by the particular choice of $|\Phi\rangle$. Indeed, phase transitions may often be detected within this CCM description by observing (for fixed $|\Phi\rangle$ ) the onset of "excited" states of negative excitation energy from Eqs. (15) - (16), as some parameter characterizing the system is varied beyond some critical value. This point is discussed more fully elsewhere [83].

The excited-state Schrödinger equation:

$$
H\left|\Psi_{\lambda}\right\rangle=E_{\lambda}\left|\Psi_{\lambda}\right\rangle \equiv\left(E+\varepsilon_{\lambda}\right)\left|\Psi_{\lambda}\right\rangle
$$

may now be combined with its ground-state counterpart to derive the CCM eigenvalue equation:

$$
e^{-S}\left[H, X^{\lambda}\right] e^{S}|\Phi\rangle=\varepsilon_{\lambda} X^{\lambda}|\Phi\rangle,
$$

for the excitation energy $\varepsilon_{\lambda} \equiv\left(E_{\lambda}-E\right)$ directly. We may now simply write down a coupled set of homogeneous linear eigenvalue equations for the excited-state configuration coefficients $\left\{x_{I}^{\lambda}\right\}$ of the linked-cluster operator $X^{\lambda}$, by taking the inner products of Eq. (18) with each member of the complete set $\left\{C_{I}^{\dagger}|\Phi\rangle ; I \neq 0\right\}$. We note that the left-hand side of Eq. (18) again has the form of a similarity transform, which may be expanded by analogy with Eq. (14). In this way we observe that the excited-state CCM equations may rather simply be derived from their ground-state counterparts by replacing each multinomial term in the coefficients $\left\{s_{I}\right\}$ arising from the expansion of the left-hand side of Eq. (13), with a corresponding set of terms in which each single coefficient $s_{J}$ is replaced one at a time in turn by the corresponding factor $x_{J}^{\lambda}$, and where the zeroth-order (inhomogeneous) terms in $\left\{s_{I}\right\}$ are dropped.

In order to implement the above CCM schemes for ground-state and excitation energies, it is necessary to truncate the corresponding sets of equations. There are many ways of doing this, but the simplest is probably the $\operatorname{SUB}(n)$ scheme for the ground state, or the more general $\operatorname{SUB}(m, n)$ scheme for excited states also. In the latter case, all configurations $\left\{x_{I}^{\lambda}\right\}$ and $\left\{s_{I}\right\}$ which describe clusters of more than $m$ and $n$ particles (or particle-hole pairs in the case of number-conserving Fermi systems) respectively are set to zero. The remaining equations, derived as described from Eqs. (13) and (18) by taking their respective inner products with the wavefunctions, $C_{I}^{\dagger}|\Phi\rangle$, of the configurations retained, are then solved exactly (or as accurately as possible). Thus, for example, the SUB(3) approximation for the ground state is precisely what we earlier referred to as the CCSDT approximation. Any such ground-state approximation results in a set of parameters $\left\{s_{I}\right\}$ which may then be used as input to the excited-state 
equations. It is clear that in the $\operatorname{SUB}(m, n)$ approximation the excitation energies $\left\{\varepsilon_{\lambda}\right\}$ are equivalently obtained by diagonalization of the operator $\left(e^{-S} H e^{S}-E\right)$, where $S$ is the SUB $(n)$-approximated CCM ground-state correlation operator, within some subspace of multiconfigurational states defined by the truncation index $m$.

In general, there is no reason at all why the truncation indices $m$ and $n$ should not be chosen differently. This freedom allows a considerable degree of flexibility within the method. Further work in this connection [84] has shown that by further imbedding the theory of linear response within the CCM, each of the usual energy-weighted-moment sum rules for the dynamic (liquid) structure function may, in principle, be exactly decomposed into an infinite cluster-hierarchy of sub-sum-rules. One outcome of this development is a rather valuable bridge between the ground-state and excited-state CCM formalisms which, although clearly intimately related, are otherwise somewhat disjoint in terms of their separate truncation systematics.

The above work [84] also gives a set of independent exact yardsticks for the linked-cluster amplitudes, against which approximation schemes in both ground and excited formalisms can be tested for mutual compatibility. Furthermore, it provides a mechanism whereby any additional knowledge of the system (obtained from either experiment or alternative theoretical formalisms) about either the excitation spectrum or the ground-state correlations, may be used to extract information within the CCM on the other. Finally in this context, it is interesting to note that by making the simple approximation that the lowest members of the CCM sub-sum-rules are saturated by a single (collective or "giant resonance") state, we regain the important Biji-Feynman relation [85] for the excitation spectrum in terms of the static structure function. We believe that this aspect of the CCM may play a rather important formal role in the future developments.

Physically, one might intuit that the above single-reference version of the CCM is most likely to be a good approach for "closed-shell" systems, for which there is an essentially unique choice of model state $|\Phi\rangle$. Conversely, we turn now to "open-shell" systems for which, a priori, a multireference approach appears to provide a more reasonable calculational framework. We argue by analogy with the single-reference ground-state CCM approach discussed above. Thus, it is clear that the single-reference ground-state scheme may be viewed at a rather shallow level as simply providing, in any particular approximation scheme, a corresponding (and, in fact, very sophisticated and clever) partial resummation of an infinite set of terms (or Goldstone diagrams) in the nondegenerate version of TIPT. Indeed, our basic Eq. (13) may be viewed in this light as an embodiment of the historically important linked cluster theorem of Goldstone [86] for the energy.

In the same spirit, Brandow [87] first showed that in the intermediate normalization scheme there exists a similar linked diagram expansion (or "linked valence expansion") in the degenerate version of TIPT. This was the first such formulation which was both size-extensive and size-consistent, and Brandow showed how it was necessary for his formulation to be given in terms of a so-called "complete model space". This was defined to be one in which the multireference model state contained all possible $N$-body configurations (or Slater determinants for the usual number-conserving fermionic case) that can be formed by distributing the valence particles among a selected set of valence orbitals. Brandow further showed how his perturbative expansion could be expressed diagrammatically in terms of so-called "folded diagrams". Several authors have since described how to embody and extend this degenerate version of TIPT within the CCM 


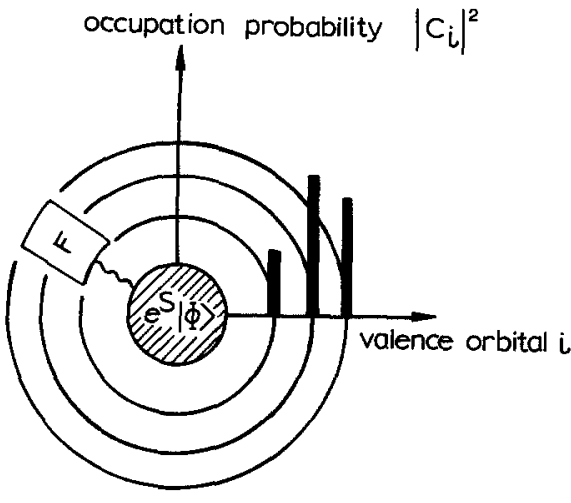

Fig. 2. A schematic representation of the essential elements of the multireference coupled cluster parametrization of the ket-state energy eigenfunctions of an open-shell system

framework, via a multireference approach. Perhaps the earliest within the physical (as opposed to chemical) context were the Bochum group of Kümmel, Offermann and Ey [88]. Many other variants exist, most of which have been especially developed for use in quantum chemistry, but for the present overview purposes I describe very briefly only this version. Its essential ingredients are indicated schematically in Fig. 2.

Thus, we start with a closed-shell system of $N$ particles (whose CCM singlereference model state is now indicated as $\left|\Phi_{N}\right\rangle$, and whose exact ground-state energy is $E \equiv E_{N}^{0}$ ), and add valence particles (or holes) one at a time. The basic idea is to keep the closed shell as a starting wavefunction, and to incorporate specifically into it the extra correlations from adding the valence particles. If we now denote the single-particle creation operators as $\left\{a_{\alpha}^{\dagger}\right\}$, we may distinguish three sorts of single-particle states, namely: (i) orbitals occupied in $\left|\Phi_{N}\right\rangle$ (labelled $\alpha \rightarrow \mu, v, \ldots$ ); (ii) valence orbitals (labelled $\alpha \rightarrow i, j, \ldots$ ) partially occupied by the valence particles outside the core; and (iii) the remaining "unoccupied" orbitals (labelled $\alpha \rightarrow \varrho, \sigma, \ldots$ ). The multireference CCM ansatz for the exact $(N+1)$-particle states is given in the form:

$$
\left|\Psi_{N+1}^{\alpha}\right\rangle=\sum_{i}^{\gamma} e^{S}\left[1+F^{(1)}\right] a_{i}^{\dagger}\left|\Phi_{N}\right\rangle C_{i}^{\alpha},
$$

where $S$ is assumed known from the $N$-body "closed-shell" calculation, and where the sum on $i$ runs over the set $\mathscr{V}$ of valence orbitals considered as actually degenerate or quasidegenerate. Thus, the states $\left\{a_{i}^{\dagger}\left|\Phi_{N}\right\rangle ; i \in \mathscr{V}\right\}$ form a set of multireference $(N+1)$-body Slater determinants for the set of low-lying states (labelled $\alpha$ ) which we wish to construct.

Whereas the coefficients $\left\{C_{i}^{\alpha}\right\}$ determine the mixture of uncorrelated states in the multireference model state, the operator $F^{(1)}$ now describes the dressing of the bare valence particle by its interactions with the core. Thus, we have the decomposition:

$$
F^{(1)}=\sum_{n=1}^{N+1} F_{n}^{(1)},
$$

where, for example, $F_{1}^{(1)}$ describes the one-body (Hartree-Fock) part of the valence problem:

$$
F_{1}^{(1)}=\sum_{\varrho} \sum_{i}^{\mathscr{V}}\left\langle\varrho\left|F_{1}^{(1)}\right| i\right\rangle a_{\varrho}^{\dagger} a_{i}
$$


and $F_{2}^{(1)}$ describes the "core polarization" terms which arise from the correlations between the valence particle and any one core particle:

$$
F_{2}^{(1)}=\frac{1}{2} \sum_{\eta_{1}, \eta_{2}} \sum_{v} \sum_{i}^{r}\left\langle\eta_{1} \eta_{2}\left|F_{2}^{(1)}\right| i v\right\rangle_{A} a_{\eta_{1}}^{\dagger} a_{\eta_{2}}^{\dagger} a_{v} a_{i},
$$

where the labels $\eta_{1}$ and $\eta_{2}$ indicate any extra-core state (i.e., valence or "unoccupied"). We note that the CCM ansatz of Eq. (19) is completely general provided only that, as in the single-reference counterpart of Eq. (10), the states $\left|\Psi_{N+1}^{\alpha}\right\rangle$ do not have zero inner product with all of the wavefunctions $\left\{a_{i}^{\dagger}\left|\Phi_{N}\right\rangle\right\}$ in the model space.

The comparable ansatz for the two valence-particle $(N+2)$-body wavefunctions is given as:

$$
\left|\Psi_{N+2}^{\beta}\right\rangle=\sum_{i, j}^{\mathscr{V}} e^{S}\left[1+F^{(1)}+\frac{1}{2}: F^{(1)^{2}}:+F^{(2)}\right] a_{i}^{\dagger} a_{j}^{\dagger}\left|\Phi_{N}\right\rangle C_{i j}^{\beta},
$$

where the factor of $\frac{1}{2}$ in the quadratic term describing two "dressed" but uncorrelated valence particles prevents us, as usual, from counting each excitation twice; and this term is also normal-ordered so as to avoid contractions (or links) between them, which are more properly contained in the genuine twovalence-particle-plus-core correlation operator $F^{(2)}$ :

$$
F^{(2)}=\sum_{n=2}^{N+2} F_{n}^{(2)}
$$

If we proceed further in this fashion to add an arbitrary number of valence particles outside the core, we rapidly arrive at the normal-ordered exponential ansatz (where the normal-ordering is always performed with respect to an appropriate closed-shell system or core) first written down explicitly by Lindgren [89], although the formulation of Ey [88] is equivalent.

By inserting Eqs. (19) and (23) into the respective $(N+1)$-body and $(N+2)$ body Schrödinger equations and premultiplying as usual by the factor $e^{-S}$, it is relatively straightforward to derive equations for the energy eigenvalues $E_{N+1}^{\alpha}$ and $E_{N+2}^{\beta}$. Suitable projections into the model space thus lead to secular equations for the coefficients $C_{i}^{\alpha}$ and $C_{i j}^{\beta}$. In fact, it is not difficult to show (and see, e.g. Refs. $[23,24,88]$ ) that these may be represented as generalized eigenvalue equations for fully-linked one- and two-body effective Hamiltonians respectively (which yield the folded diagrams of MBPT), with 'eigenvalues' equal to the respective excitation energies. For example, in the single-valence case, this eigenvalue is $\varepsilon_{\alpha} \equiv E_{N+1}^{\alpha}-E_{N}^{0}$. Similarly, by projecting out of the model space onto "unoccupied" states, we derive the equations which determine the matrix elements of the operators $F^{(1)}$ and $F^{(2)}$. The interested reader is referred to the literature cited above for further details.

Until fairly recently, the CCM and the associated linked diagrammatic expansions have been restricted to complete model spaces and the intermediate normalization scheme. The main disadvantage of complete model spaces is that even for a problem restricted to relatively few single-particle orbitals, the dimensionality of the space can be prohibitively large for practical calculations. Mukherjee [90] first showed that for general incomplete model spaces, the condition that the CCM cluster operators and the effective Hamiltonian are both connected, is normally incompatible with out previous choice of intermediate normalization. Conversely, by abandoning this normalization, these connectivity properties may be reinstated. A more detailed discussion of these points would 
take us too far afield for present purposes however, and the interested reader is referred instead to the literature [91].

Up to this point we have parametrized only the ket energy eigenstates of the system, and we have made use of the Schrödinger equation itself to extract the energy eigenvalues without the need to consider the bra states. However, when we extend the discussion to arbitrary properties of the system, we cannot avoid the use of the bra states to form expectation values. As Fig. 1 indicates schematically, it is at this point that the overall CCM formalism now divides into the so-called normal (NCCM) and extended (ECCM) schemes [74]. (Quantum chemists should beware that this latter terminology has sometimes also been used in the past to indicate a truncated NCCM calculation that goes beyond the CCSD approximation.) The crucial feature of both CCM formulations is that the corresponding bra and ket states are parametrized independently in a way that does not explicitly preserve their Hermitian adjoint relationship to each other, and which as a consequence actually breaks it at a general (e.g. SUB $(n)$ scheme) level of truncation. The underlying reasons for this have been well described elsewhere [74, 75, 80], and are briefly reviewed below.

In the first place, the most direct expression for the expectation value $\vec{A}$ of an arbitrary operator $A$, in an energy eigenstate whose ket form is specified in the single-reference CCM formulation by Eq. (10), is clearly given by:

$$
\begin{aligned}
\bar{A} & =\left\langle\Phi\left|e^{S^{\dagger}} A e^{S}\right| \Phi\right\rangle \mid\left\langle\Phi\left|e^{S \dagger} e^{S}\right| \Phi\right\rangle \\
& =\left\langle\Phi\left|\left(e^{S \dagger} A e^{S}\right)_{\mathscr{L}}\right| \Phi\right\rangle .
\end{aligned}
$$

The suffix $\mathscr{L}$ in Eq. (25b) indicates that this expression is again confined to a sum of linked diagrams [5, 92]. Nevertheless, when expanded in powers of $S$ and $S^{\dagger}$, Eq. (25b) is of infinite order, unlike the corresponding expansion of Eq. (14) which terminates after a finite number of terms. Furthermore, if the resulting expansion of Eq. (25b) is approximated by truncation at any given order, the resulting expression no longer has the property of agreeing with the HellmannFeynman theorem [93] exhibited by the exact energy eigenstate. In turn, this important theorem implies that $\bar{A}$ should be calculated diagrammatically from the same set of Goldstone diagrams for the energy (as summed by the CCM at any level of truncation, for example), but in which each interaction potential is replaced in turn by the operator $A$, as has been pointed out by Thouless [94]. A more sophisticated method of calculating $\bar{A}$ within the CCM due to Kümmel [95] also conflicts with the Hellmann-Feynman theorem.

Monkhorst [96] first formulated a CCM prescription for $\bar{A}$ which is compatible with the Hellmann-Feynman theorem, by employing techniques of linear and higher-order response theory. It is interesting to note, however, that he never explicitly introduced a functional form for $\bar{A}$. Once supplemented [97] by the basis set effects that are needed for the practical analytical evaluation of energy derivatives within quantum chemistry, the scheme of Monkhorst was successfully applied [98] to the prediction of vibrational spectra and to the location of transition states for decomposition reactions.

In this context, Arponen [74] introduced two distinct CCM parametrizations of the bra state. Neither is manifestly the Hermitian conjugate of the corresponding ket state, but both have the following advantages which more than compensate for this loss, namely: (i) they lead to explicit average-value functionals $\bar{A}$; (ii) they are compatible with the Hellmann-Feynman theorem; and (iii) they are derivable from a variational principle (although the reader should beware that 
the loss of the Hermitian-adjoint relation between bra and ket states ensures that the upper bound property for energies is also lost).

The NCCM is basically a generalization of the method of Monkhorst [96]. The bra ground-state wavefunction $\langle\tilde{\Psi}|$ corresponding to $|\Psi\rangle$, where:

$$
\langle\tilde{\Psi}| H=E\langle\tilde{\Psi}|,
$$

is the counterpart to Eq. (12), is parametrized in the NCCM as:

$$
\langle\tilde{\Psi}|=\langle\Phi| \tilde{S} e^{-S}
$$

Equations (10) and (27) characterize the NCCM, together with Eq. (11) for the operator $S$ and its counterpart:

$$
\tilde{S}=1+\sum_{I}^{\prime} \tilde{s}_{I} C_{I}
$$

for $\tilde{S}$, which asserts that $\tilde{S}$ is constructed in terms of destruction operators only (with respect to $|\Phi\rangle$ ). The choice of unity for the constant term in Eq. (28) implies the manifest normalization, $\langle\tilde{\Psi} \mid \Psi\rangle=\langle\Phi \mid \Phi\rangle=1 \quad(=\langle\Phi \mid \Psi\rangle)$. The ground-state version of the NCCM is now completely specified by the set of parameters $\left\{s_{I}, \tilde{s}_{I}\right\}$, and in particular an arbitrary expectation value $\bar{A}$ may be written in the NCCM as:

$$
\bar{A}=\left\langle\Phi\left|\tilde{S e}^{-S} A e^{S}\right| \Phi\right\rangle=\bar{A}\left[s_{I}, \tilde{s}_{I}\right] .
$$

It is important to realize that the coefficients $\left\{s_{I}, \tilde{s}_{I}\right\}$ are regarded as independent (variational) parameters, although formally we have the relation:

$$
\langle\Phi| \tilde{S}=\frac{\langle\Phi| e^{S^{\dagger}} e^{S}}{\left\langle\Phi\left|e^{S^{\dagger}} e^{S}\right| \Phi\right\rangle} .
$$

The coefficients $\left\{s_{I}, \tilde{s}_{I}\right\}$ are themselves determined by requiring the expectation value (29) for the Hamiltonian, $\bar{H}$, to be stationary with respect to all independent variations:

$$
\begin{aligned}
& \frac{\delta \bar{H}}{\delta \tilde{s}_{I}}=0 \Leftrightarrow\left\langle\Phi\left|C_{I} e^{-S} H e^{S}\right| \Phi\right\rangle=0, \quad I \neq 0, \\
& \frac{\delta \bar{H}}{\delta s_{I}}=0 \Leftrightarrow\left\langle\Phi\left|\tilde{S} e^{-S}\left[H, C_{I}^{\dagger}\right] e^{S}\right| \Phi\right\rangle=0, \quad I \neq 0 .
\end{aligned}
$$

Equations (31a) are precisely the previous CCM equations for the ket-state coefficients $\left\{s_{I}\right\}$. Since the states $\left\{C_{I}^{\dagger}|\Phi\rangle\right\}$ are complete over the many-body Hilbert space, they are equivalent to Eq. (13). Equations (31b) are a coupled set of linear equations for the new NCCM coefficients $\left\{\tilde{s}_{I}\right\}$, in terms of given $\left\{s_{I}\right\}$. We note also that the ground-state energy calculated from the stationary point of $\bar{H}=\left\langle\Phi\left|\tilde{S} e^{-S} H e^{S}\right| \Phi\right\rangle$, is identical, at any level of approximation, to our earlier CCM result $E=\left\langle\Phi\left|e^{-S} H e^{S}\right| \Phi\right\rangle$, due to Eqs. (31a) being satisfied.

Although the NCCM expression, $\bar{A}=\bar{A}\left[s_{I}, \tilde{s}_{I}\right]$, is generally composed wholly of linked terms, it is easy to see that (unlike the operator $S$ ) the new operator $\tilde{S}$ itself contains unlinked terms. On the other hand, it was also shown [74] that $\tilde{S}$ could again be written in exponential form:

$$
\tilde{S}=\exp (\tilde{\Sigma}) ; \quad \tilde{\Sigma}=\sum_{I}^{\prime} \tilde{\sigma}_{I} C_{I}
$$

in terms of a new destruction operator $\tilde{\Sigma}$ which contains only linked pieces. The corresponding complete parametrization of $\bar{A}$ in terms of the operators $S$ and $\tilde{\Sigma}$ 
constitutes the ECCM. Nevertheless, for many purposes it is more convenient to introduce also a new creation operator $\Sigma$, such that:

$$
\Sigma|\Phi\rangle=(1-|\Phi\rangle\langle\Phi|) e^{\tilde{\Sigma}} S|\Phi\rangle ; \quad \Sigma=\sum_{I}^{\prime} \sigma_{I} C_{I}^{\dagger},
$$

with corresponding inverse transformations:

$$
\sigma_{I}=\left\langle\Phi\left|C_{I} e^{\tilde{\Sigma}} S\right| \Phi\right\rangle, \quad s_{I}=\left\langle\Phi\left|C_{I} e^{-\tilde{\Sigma}} \Sigma\right| \Phi\right\rangle .
$$

Either of the sets $\left\{s_{I}, \tilde{\sigma}_{I}\right\}$ or $\left\{\sigma_{I}, \tilde{\sigma}_{I}\right\}$ is complete and sufficient to specify fully the ECCM description of the ground state. Each member of both sets is fully linked, but the latter set is formally more useful since it provides a set of variables which are canonically conjugate in some well-defined sense [75], just as also does the NCCM set $\left\{s_{I}, \tilde{s}_{I}\right\}$.

One of the key distinguishing features of the ECCM which follows from its double exponential structure (and hence also its double similarity transform structure, and the subsequent double-linked [74] nature of its diagrams), is that by contrast with the NCCM and the CI method, all of the basic amplitudes that completely characterize the system are linked-cluster quantities with well-defined diagrammatic representations [74]. In turn, they all therefore obey the very important cluster property, namely that they become asymptotically zero as any subset of particles described by the amplitude becomes far removed from the remainder, in real space. The entire system may thus be parametrized in terms of a complete set of ECCM multilocal, classical (i.e., $c$-number) amplitudes. It has also been shown [80] how an arbitrary quantum-mechanical problem with underlying Schrödinger dynamics is thereby exactly mapped onto a classical field theory, in which the ECCM amplitudes $\left\{\sigma_{I}, \tilde{\sigma}_{I}\right\}$ interact via nonlocal classical interactions.

Extensions of the ECCM have been made [75,99] to consider both excited states and general (nonstationary) dynamical behaviour, in the latter case by formulating an analogous variational principle for the action. It has thus been demonstrated how the amplitudes $\left\{\sigma_{I}, \tilde{\sigma}_{I}\right\}$ may also be viewed as generalized many-body mean fields or quasilocal order parameters, by considering their small-amplitude oscillations around a stationary equilibrium point. The overall structure of the ECCM is indicated schematically in Fig. 3, and the interested reader is referred to the literature $[74,75,80,99-104]$ for further details. We note here only that the ECCM is, to the best of our knowledge, unique as a formulation of quantum many-body theory in which every fundamental amplitude exactly obeys the cluster property at all levels of approximation. It is clear that only such formulations have the possibility to describe both the local properties of many-body systems and such global properties as their phase transitions, states of topological excitation or deformation, spontaneous symmetry breaking, and general nonequilibrium behaviour.

Referring back to Fig. 1, we have now completed our overview of the main formal aspects of $\mathrm{CC}$ theory as it currently exists for pure states. We mention in conclusion that an extension to mixed states (of relevance to systems at nonzero temperatures) has also been given [105], in terms of the Bloch equation for the statistical density operator.

We turn in the remainder of this paper to a survey of the main applications of the CCM techniques described in this Section, and summarized pictorially in Fig. 1. 


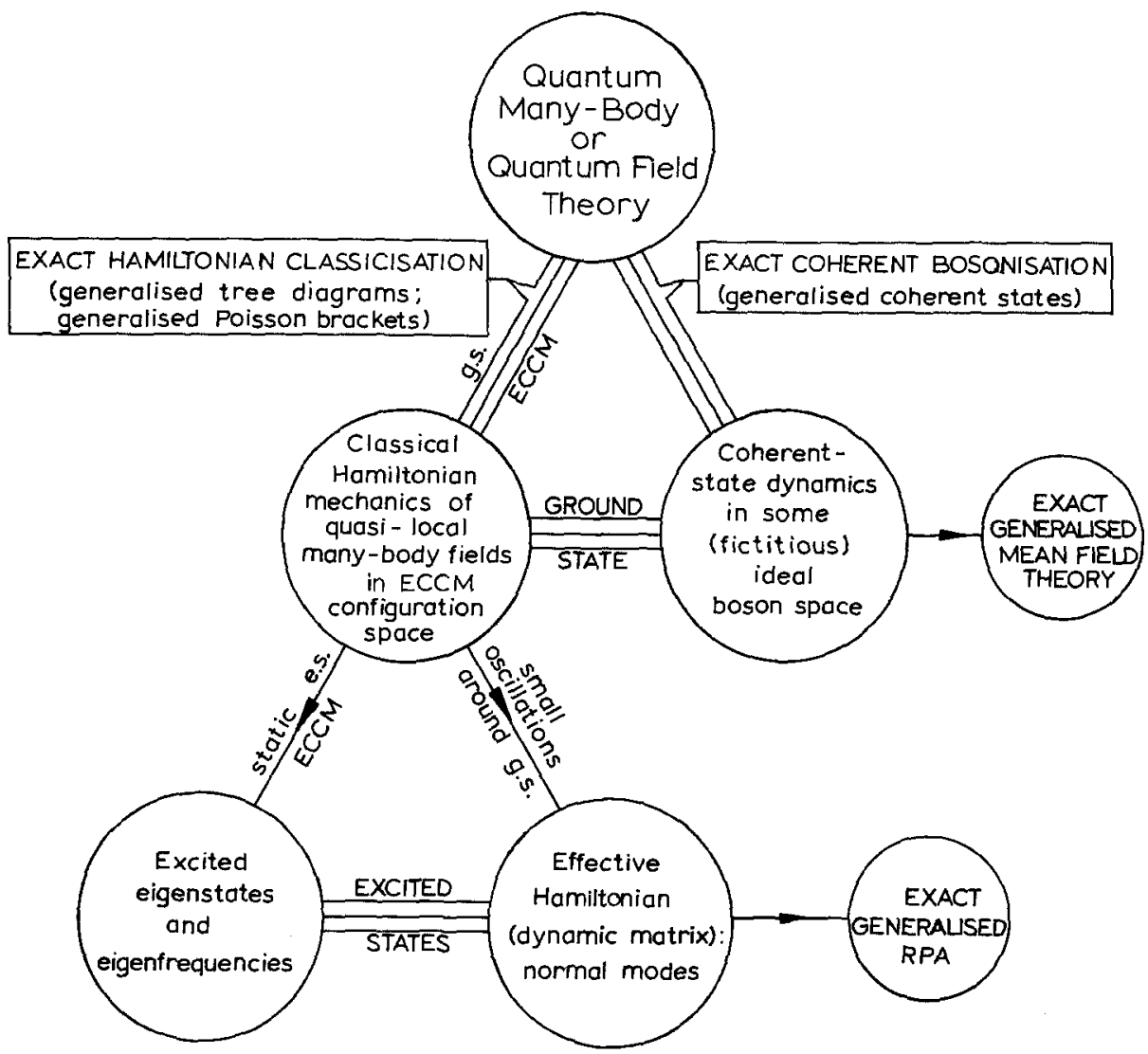

Fig. 3. A schematic representation of the hierarchical structure and the most general features of the extended coupled cluster method for quantum many-body and quantum field theory

\section{CC applications in nuclear physics}

The collection of naturally occurring atomic nuclei exhibits an exceptionally rich diversity of quantum many-body phenomena. In principle, they therefore provide the many-body theorist with a unique laboratory of strongly-interacting systems, on which he may test the various microscopic, universal tools at his disposal. Furthermore, the nuclear theorist hopes that such studies may provide valuable insights into the nature of the strong interaction itself. Unfortunately, this rather clearly defined picture has, in practice, been much blurred. At the heart of the underlying problem lies the fact that the internucleonic interaction is not uniquely known. Indeed, whereas for atoms and molecules the interelectronic forces are almost wholly Coulombic (with relativistic corrections and other small corrections from quantum electrodynamics, QED), in nuclear physics there is no well-defined potential at all.

At the most fundamental level of our present understanding, the internucleonic forces must derive from the quantum chromodynamics (QCD) of the basis quark and gluon fields. Just as the interatomic forces between neutral atoms are 
the relatively complicated "remnant forces" of the underlying Coulombic forces between their charged constituents, so the very complex nucleon-nucleon (NN) force between the "colourless" nucleons is presumed to be the remnant force of what may be a much simpler force given by QCD between their constituent "coloured" quarks. At a less deep level, we may also model the forces between nucleons as being mediated by meson-exchange processes. The presumption is that this description suffices so long as we do not probe the nucleus at energies high enough or at distances short enough to uncover the quarkic content of the baryons and mesons. Thus, at this level, nuclear theory should deal with a consistent quantum field theory of (at least) nucleons and mesons. However, such multi-meson exchange processes are ill-understood, and the usual versions of such field theories are fraught with problems concerning renormalizability. Thus, with very few exceptions (and see, for example, the later discussion in Sect. 7.2 for a particularly noteworthy CCM contribution), such calculations are not directly undertaken in nuclear physics.

Instead, it has been almost universal practice since the earliest days of nuclear physics to model the interaction forces between collections of nucleons by static, few-body (and, typically, only two-body) potentials. Therefore, one of the most urgent questions in nuclear physics has been whether the nuclear forces inside nuclei are indeed well represented by a sum of two-body potential interactions. A more accurate statement of the question would lead us to enquire whether there are many-body effects that even the best two-body potentials cannot account for. Undoubtedly, the implicit hope and expectation was that two-body potentials would indeed suffice at least for the ground-state and low-lying energy levels of nuclei, and that many other common properties of them could also be similarly explained in broad qualitative outline, if not in fine quantitative detail.

In order sensibly to pose the above question, one needs first to be able to construct reasonable and accurate representations of the two-nucleon (and, if necessary, the three-nucleon, etc.) force. Secondly, in order to answer it, one needs to have accurate many-body theories which can handle such forces with confidence. We consider these two points in turn. It is clearly a prerequisite of any NN potential used that it should fit all of the available data on $\mathrm{NN}$ scattering and on the known two-body bound state, namely the deuteron. Naturally, these data are subject to experimental errors, as well as being incomplete. For example, the scattering data extend only up to some maximum value of the relative momentum in a given partial-wave channel, and only in partial waves up to some maximum angular momentum.

More importantly, even if the two-body (scattering and bound-state) data were complete, the quantum-mechanical inversion process to reconstruct the potential from them is not unique. Only when such additional assumptions as that the potential is local are made, may the potential be uniquely determined in principle. Thus, for example, both a local potential and a one-term nonlocal but separable potential can be chosen (in a given partial-wave channel, say), so that they are indistinguishable in terms of such on-energy-shell two-body scattering data as the phase shift in that channel as a function of relative momentum. Comparable uncertainty may be couched in terms of whether the potential should be local but energy-dependent, for example. Additional complications concern the short-range repulsion. It is difficult from experimental data alone to distinguish between hard-core potentials and ones with a softer, but still strong, core. Indeed, without being unduly radical, it is even possible to contemplate 
potentials which have no perceptible short-range repulsion (or which are even attractive at short distances), at the expense of introducing energy dependence of a particular form, for example [106].

There is hence considerable uncertainty over the extraction of the NN potential, and this basically arises from the fact that there is no simple fundamental theory for it. What is clear from the various good fits to the experimental data, is that the potential is just about as complicated as is permitted by the various invariance laws and symmetry principles. For example, it differs in every partial wave, and in the spin-triplet channels it takes full advantage of the allowed possibilities to depend on the spin coordinates. This dependence is often modelled for a specific spin-triplet channel in terms of a force containing both a (Wigner) central term as well as such spin-dependent components as a tensor force. It is important for present purposes to note that these complexities in the NN force cause no problem either in principle or in practice for CCM techniques. By contrast, many of the variational and CBF methods that have been implemented for simple central forces, founder when faced with such more complicated potentials.

To summarize our discussion so far, there exist several quite different phenomenological NN potentials which provide equally valid excellent fits to the two-body data. On this basis (namely, on-energy-shell results) they cannot be distinguished. On the other hand, they differ in their off-energy-shell properties, and hence in their predictions for the properties of nuclei with mass number $A>2$. Nevertheless, in order to compare their predictions for heavier nuclei it is clearly necessary to have a many-body theory whose results for these stronglyinteracting systems are believed to be converged and accurate, and, ideally, are demonstrably so. The theoretical situation is succinctly but accurately described by saying that of the various techniques available (and described in Sect. 2), only the CCM has provided results of such quality that one can assert on that basis that no known two-body potential can properly describe even the ground-state saturation properties, let alone the low-lying excitation spectra, of a range of closed-shell nuclei $\left({ }^{4} \mathrm{He},{ }^{16} \mathrm{O},{ }^{40} \mathrm{Ca}\right)$, and their open-shell neighbours formed by adding one or two valence particles or holes.

Furthermore, we note that these CCM calculations have for the most part been available for ten years or so, and in the meantime no other microscopic technique has, to the best of our knowledge, been able to demonstrate comparable power and accuracy in this field. Indeed, even now, most of the alternative calculations still being performed on open-shell nuclei, using, for example, the degenerate versions of MBPT and the associated technology of folded diagrams, contain only a (small, and often inconsistent or invalid) subset of the terms included by the demonstrably converged results of the Bochum group $[23,24,81,107]$. With hindsight it is clear that these early illustrations of the power and accuracy of the CCM were at least a decade ahead of their time.

Now, we know that in principle there are extra three-body effects (from meson-exchange) in the three-nucleon problem that cannot be represented by the sum of two-body effects. We might therefore be tempted to introduce suitably parametrized three-body potentials to model these effects. Several such attempts have indeed been made, although it is probably fair to say that in practice insufficient data exist to do this convincingly, particularly in view of the theoretical uncertainty which also surrounds this area. Although the incorporation of $n$-body forces with $n=3,4, \ldots$, poses none other than technical problems (at least for the CCM), the whole procedure only truly makes sense if 
the process converges rapidly. In fact, so long as the theoretical tools used in nuclear many-body physics were not of high enough precision or, what is the same thing, so long as the approximations had not converged, one could still cling to the hope that two-body potentials might suffice to explain the experimental situation, at least at not too high an energy scale.

The great merit of all of the highly sophisticated CCM calculations on nuclei (and see, e.g., Refs. [23, 24] for reviews) that have by now been performed, is that just because they fail to reproduce even the low-energy data, and because for the first time has a many-body method achieved high enough precision to be certain of the accuracy of the results, so can it now be claimed with confidence that nuclei are not many-body systems interacting via two-body forces. (Indeed, the inclusion of the best of the then available three-body potentials did not markedly improve the fit with experiment [23, 24].) Of course, in absolute terms this has been known for a long time, but what is important is that for the first time the CCM calculations have demonstrated that this simple idealized picture of nuclei will not serve as an adequate model for even the simplest ground-state structure. Many such examples are given in Refs. [23, 24].

We conclude this discussion on applications to finite nuclei by briefly mentioning some of the relevant technical details of the CCM calculations in this field. One of the most important concerns the hard-core nature of the NN interaction, which ensures that at least some part of the matrix elements of the two-body potential becomes infinite, in any complete single-particle basis. Finiteorder Goldstone diagrams are thus ill-defined, and partial summations need to be carried out to infinite order. Within conventional MBPT this is achieved by re-expressing the perturbation series in terms of the "dressed" BruecknerBethe-Goldstone $G$-matrix [18-20], in place of the bare potential $V$. Each bare interaction is thereby replaced by an infinite ladder sum over two-body terms which represent repeated scattering of the pair. The rest of the many-body medium serves only to exclude intermediate scattering states which are already "occupied" (in accord with the Pauli exclusion principle), and possibly also to provide a self-consistent one-body potential in which the scattering pair moves.

Certainly this basic idea of grouping interaction lines into ladder series can also be achieved within the CCM. Indeed, such diagrammatic structures are generated automatically, at even the lowest SUB(2) level of approximation. Where problems do arise in such truncations as the $\operatorname{SUB}(n)$ scheme is that the replacement of a bare potential everywhere in the diagrams so generated by iteration of the approximated CCM equations, can lead to diagrams which are not included at the same level of truncation. This actually occurs in both the NCCM and ECCM formulations at every SUB $(n)$ level, and it thereby ensures that approximated cluster operators $S_{n}$ are not well-defined for hard-core potentials in the standard $\operatorname{SUB}(n)$ truncations.

The cure, however, is simple. It leads to the so-called (hard-core) $\operatorname{HCSUB}(n)$ scheme (otherwise known as the $\chi_{n}$-truncation or the Bochum truncation scheme). Thus, at a fixed ordinary $\operatorname{SUB}(n)$ level, one first identifies that subset of terms which when iterated together leads only to diagrams which are still contained in this original particular $\operatorname{SUB}(n)$ class when each bare interaction $V$ is replaced by a ladder-summed $G$-matrix, and when the relative time-orderings of the remaining interactions are kept fixed. The related $\operatorname{HCSUB}(n)$ approximation scheme is closely related to the hole-line expansion of Bethe. It is discussed further elsewhere $[23,24,74]$. The CCM calculations on the closed-shell nuclei, for example, have been performed up to the HCSUB(4) level, and the two- and 
three-body terms in the resulting equations have been treated exactly, whereas in the rather complicated four-body terms only their most important contributions have been kept. Although this latter approximation is not without danger, the resulting $\operatorname{HCSUB}(n)$ sequence of ground-state energy approximants for $n \leqslant 4$ shows rapid convergence for all nuclei and for all choices of NN potential that have been studied.

Very similar calculations have also been performed for infinite nuclear matter [108]. Technically, this is a more complicated system than finite nuclei, since the latter have a relatively large surface region and hence a lower average density. Within the CCM this is reflected by the fact that the three- and four-body cluster correlations are relatively larger in nucler matter, and the $\operatorname{HCSUB}(n)$ sequence is hence somewhat slower to converge. Nevertheless, the overall quality of the results is compatible with those obtained in finite nuclei. There is again disagreement between the "experimental" saturation density and energy as extrapolated from the semiempirical mass formula, and the converged results obtained by using any of the reasonable phenomenological NN potentials. The CCM results also agree for a given potential with those obtained using the best available variational techniques in this case (and see, e.g., Ref. [108]).

Another important technical point in these nuclear calculations concerns the choice of single-particle orbitals. For such homogeneous infinite systems as nuclear matter there is no choice to be made since the requirement of translational invariance implies that the one-body partition of the cluster correlation operator vanishes identically, $S_{1}=0$. For finite nuclei a common choice is to use a harmonic oscillator basis with some particular choice of the ("natural frequency") oscillator parameter. Another choice has been the so-called "maximum overlap" orbitals (which are often denoted as Brueckner orbitals in quantum chemistry), in which $S_{1}=0$ is a necessary condition. They are simply the solutions to the one-body CCM equation obtained from Eq. (13) by projecting onto the $1 p-1 h$ basis, with $S_{1}=0$. In $\operatorname{SUB}(1)$ approximation this is simply the Hartree-Fock condition, but in higher-order truncations the two- and threebody correlations generated by $S_{2}$ and $S_{3}$ have some effect on these states. Other choices of single-particle orbitals include the so-called "natural orbitals", defined to be the eigenstates of the one-body density matrix.

For all (light and medium-light) finite nuclei studied, the results are rather insensitive to any reasonable choice of basis. Indeed, the maximum-overlap orbitals are very close to harmonic oscillator functions, and hence the latter basis with a reasonable choice of oscillator parameter (appropriate to the average density of the nucleus) works rather well. In this sense the situation in nuclear physics is quite unlike that in quantum chemistry, where the selection of the starting single-particle orbitals is itself a major task. Presumably this is largely due to the more complicated geometry.

We note parenthetically in this regard that Kümmel [109] has also investigated the sufficiency conditions for the maximum-overlap orbitals actually to maximize the overlap of the model state $|\Phi\rangle$ with the true state $|\Psi\rangle$, rather than merely to extremize it, as given by the usual necessary condition. He shows in particular how the extra sufficiency criterion can be utilized in practice to identify within the CCM the onset of a critical "shape instability" which marks the transition to a different optimal basis, or configuration, as some appropriate internal parameter is varied.

A final technical point concerns the general fact that model reference states often violate some exact symmetry. A relevant example here is that unless the 
single-particle orbitals are plane waves, the model state $|\Phi\rangle$ is not an eigenstate of the total linear momentum operator $\vec{P}$, and hence not translationally invariant. Nevertheless, the exact nuclear ground state should be a simultaneous eigenstate of both the Hamiltonian $H$ and $\vec{P}$, since $[H, \vec{P}]=0$. Thus, we expect to be able to impose that the full CCM representation of the wavefunction given by Eq. (10) is an eigenstate of $\vec{P}$, so that we have in addition to Eq. (13):

$$
e^{-S} \vec{P} e^{S}|\Phi\rangle=\vec{K}|\Phi\rangle \text {. }
$$

On the other hand, in $\operatorname{SUB}(n)$ or $\operatorname{HCSUB}(n)$ approximations we shall not generally be able to preserve the exact translational invariance.

Most calculations in nuclear physics have dealt with the above problem by using the internal Hamiltonian:

$$
H \rightarrow H_{\text {int }}=H-T_{\mathrm{CM}},
$$

where $T_{\mathrm{CM}} \equiv \vec{P}^{2} / 2 M$ is the kinetic energy operator of the centre of mass (CM), and $M$ is the total mass. For a system of $A$ nucleons of mass $m$ each, momentum $\vec{p}_{i}$, and interacting via pairwise NN potentials $V_{i j}$, the internal Hamiltonian is thus:

$$
H_{\mathrm{int}}=\left(1-\frac{1}{A}\right) \sum_{i=1}^{A} \frac{p_{i}^{2}}{2 m}+\sum_{i<j=1}^{A}\left(V_{i j}-\frac{\vec{p}_{i} \cdot \vec{p}_{j}}{A m}\right) .
$$

The main effect of the removal of the CM energy, which is itself most important for the lightest nuclei, is thus to produce a momentum-dependent interaction term. All of the calculations done by the Bochum group [23, 24, 81, 107] noted above, have been performed using the $H_{\text {int }}$ of Eq. (37). Undoubtedly, at any level of approximation, its use is essentially a compromise between using the exact Eqs. (13) and (35) and neglecting the CM motion entirely. Fortunately, this approximation has been checked recently [76] for the ${ }^{4} \mathrm{He}$ nucleus, and has been seen to be very good even for this very light nucleus. (In general, the approximation incurred by the use of $H_{\text {int }}$ is no worse in any CCM truncation than the errors inherent in that particular approximation from the neglect of higher-order correlations.) Finally, this latter work has also shown how to incorporate the CM motion exactly into the CCM prescription at any level of approximation, so that it provides a rigorously translationally-invariant formulation. In so doing it shows how the CCM may also be expressed more directly in terms of a coordinate-space description.

\section{CC applications to one-component Coulomb plasmas}

The electron gas (or "jellium") is one of the theoretically most well-studied of all quantum many-body systems. It was originally invented to model the electrons in a metal, in a simplified form in which the ionic lattice is replaced by a uniform positive charge, as an inert neutralizing background. The two-body potential is otherwise pure Coulombic. It is given in the momentum-space representation as:

$$
V(\vec{q})=\frac{4 \pi e^{2}}{\Omega q^{2}}\left(1-\delta_{\vec{q} 0}\right)
$$

where $\Omega$ is the normalization volume. More generally, a system of $N$ identical particles (bosons or fermions), of mass $m$ and charge $e$ each, interacting via the two-body potential of Eq. (38), is denoted as the one-component Coulomb plasma. The number density, $\varrho=N / \Omega$, may be expressed in terms of the only 
independent dimensionless coupling constant, $r_{s} \equiv r_{0} / a_{0}$, which characterizes the system, namely the average interparticle spacing, $r_{0}$, in units of the Bohr radius, $a_{0} \equiv \hbar^{2} / m e^{2}$. One may also equivalently specify $\varrho$ in terms of a (what is for bosons purely fictitious) Fermi wavenumber, $k_{F}$, applicable to an unpolarized spin $-\frac{1}{2}$ system:

$$
\varrho=\left(4 \pi r_{s}^{3} a_{0}^{3} / 3\right)^{-1}=k_{F}^{3} / 3 \pi^{2} .
$$

Henceforth, we also employ units such that $\hbar=1$. We note that Eq. (39) implies the relation:

$$
k_{F} a_{0}=\left(\alpha r_{s}\right)^{-1} ; \quad \alpha \equiv(9 \pi / 4)^{-1 / 3} .
$$

It is convenient to define the ground-state energy per particle in Rydberg units as:

$$
E / N=\varepsilon\left(e^{2} / 2 a_{0}\right)
$$

and similarly to scale, as needed, any momentum variable $\vec{q}$ in units of $k_{F}$ defined in Eq. (39), $\vec{q}=k_{F} \vec{x}$.

The one-component Coulomb plasmas are interesting systems for study because they contain only one dimensionless coupling constant $r_{s}$, and because they exhibit a phase transition between the high-density (weak-coupling, $r_{s} \rightarrow 0$ ) plasma limit and the low-density (strong-coupling, $r_{s} \rightarrow \infty$ ) limit of a Wigner crystal. The quantum statistics play a crucial role at high and intermediate densities, but become less and less important in the essentially classical lowdensity limit. Furthermore, these systems are highly nontrivial for all values of $r_{s}$, due to the long-range $\left(r^{-1}\right)$ nature of the Coulomb potential.

Let us first consider the CCM treatment of the algebraically much simpler bosonic plasma. As reference state we take the zero-momentum condensate:

$$
|\Phi\rangle=(N !)^{-1 / 2}\left(b_{0}^{\dagger}\right)^{N}|0\rangle,
$$

where $|0\rangle$ is the vacuum state, and $\left\{b_{\alpha}^{\dagger}\right\}$ are a complete set of single-boson creation operators. In the plane-wave, momentum-eigenstate representation we thus have that $\alpha \rightarrow 0$ labels the sole "occupied" (and hence hole) state, and $\alpha \rightarrow \vec{q}$ $(\neq 0)$ labels the "unoccupied" particle states. The cluster correlation operator of Eqs. (10) - (11) may now be explicitly expressed as:

$$
S=\sum_{n=2}^{N} S_{n} ; \quad S_{n}=\frac{1}{n !} \sum_{\vec{q}_{1} \ldots \vec{q}_{n}} S_{n}\left(\vec{q}_{1}, \ldots, \vec{q}_{n}\right) b_{\dot{q}_{1}}^{\dagger} \ldots b_{\dot{q}_{n}}^{\dagger}\left(N^{-1 / 2} b_{0}\right)^{n},
$$

where the matrix elements in Eq. (43) are subject to the condition $\sum_{i=1}^{n} \vec{q}_{i}=0$, which arises from the assumption that the $(N \rightarrow \infty)$ ground state is that of a homogeneous (translationally-invariant) phase.

In the present case where the interaction potential is of local two-body type and with the additional constraint from Eq. (38) that $V(\vec{q}=0)=0$, it is relatively straightforward to show that the exact two-body equation for $S_{2}(q) \equiv S_{2}(\vec{q},-\vec{q})$ becomes (in the thermodynamic limit where $N \rightarrow \infty$ at fixed $\varrho$ ):

$$
\begin{aligned}
& \frac{q^{2}}{m} S_{2}(q)+T_{\mathrm{RPA}}+T_{\mathrm{CP}}+T_{\mathrm{LAD}}+\Omega \int \frac{d \vec{q}^{\prime}}{(2 \pi)^{3}} V\left(q^{\prime}\right) \\
& \quad \times\left[2 N^{1 / 2} S_{3}\left(\vec{q}, \vec{q}^{\prime},-\vec{q}-\vec{q}^{\prime}\right)+\frac{1}{2} N S_{4}\left(\vec{q},-\vec{q}, \vec{q}^{\prime},-\vec{q}^{\prime}\right)\right]=0,
\end{aligned}
$$


where

$$
\begin{aligned}
T_{\mathrm{RPA}} & =N V(q)\left[1+S_{2}(q)\right]^{2}, \\
T_{\mathrm{CP}} & =-4 \frac{E}{N} S_{2}(q), \\
T_{\mathrm{LAD}} & =\Omega \int \frac{d \vec{q}^{\prime}}{(2 \pi)^{3}} V\left(\vec{q}-\vec{q}^{\prime}\right) S_{2}\left(q^{\prime}\right),
\end{aligned}
$$

and where the ground-state energy is given by:

$$
\frac{E}{N}=\frac{1}{2} \Omega \int \frac{d \vec{q}}{(2 \pi)^{3}} V(q) S_{2}(q),
$$

Equation (44) may be rather easily written diagrammatically (and see, e.g., Ref. [110b]).

The SUB(2) approximation is obtained from Eq. (44) by setting $S_{3}$ and $S_{4}$ to zero. The remaining four terms represent respectively: (i) the kinetic energy (KE) contribution; (ii) the terms that together with KE generate precisely the random phase approximation (RPA); (iii) the terms that generate the self-consistent energy insertions on the hole lines, namely the condensate potential (CP) terms; and (iv) the terms that generate the two-boson ladder (LAD) diagrams for repeated scattering of a pair from out of the condensate. When fully expressed in terms of dimensionless variables, the $\operatorname{SUB}(2)$ equation for the bosonic Coulomb plasma becomes:

$$
\begin{gathered}
x^{2} S_{2}(x)+\frac{4 \alpha r_{s}}{3 \pi x^{2}}\left[1+S_{2}(x)\right]^{2}-2\left(\alpha r_{s}\right)^{2} \varepsilon S_{2}(x)+\frac{\alpha r_{s}}{\pi x} \int_{0}^{\infty} d x^{\prime} x^{\prime} \ln \left|\frac{x^{\prime}+x}{x^{\prime}-x}\right| S_{2}\left(x^{\prime}\right)=0, \\
\varepsilon=\frac{2}{\pi \alpha r_{s}} \int_{0}^{\infty} d x S_{2}(x) .
\end{gathered}
$$

Although the nonlinear equation (47) is readily solved numerically, it is more instructive to examine its high- and low-density limits. In the high-density limit it is trivial to show that:

$$
\varepsilon \underset{r_{s} \rightarrow 0}{\longrightarrow} Q r_{s}^{-3 / 4}+R
$$

and furthermore that only the KE and RPA terms contribute to leading order, to give the well-known exact result:

$$
Q=-\frac{16}{5}\left(\frac{3}{\pi^{2}}\right)^{1 / 4} \frac{\Gamma\left(\frac{3}{4}\right)}{\Gamma\left(\frac{1}{4}\right)} \approx-0.8031
$$

first obtained by Foldy [111]. The CP and LAD terms also contribute to next order to give a SUB(2) value for the constant $R$ of $16 / 9 \pi$. Inspection of Eqs. (44) -(45) shows that the coupling terms to $S_{3}$ and $S_{4}$ also contribute to the constant $R$ (although not to $Q$ ). A careful and detailed calculation [110c] including these terms leads to the value $R \approx 0.0280$, and an intricate rearrangement of terms shows that this result is identical to the first correct result reported, namely that of Brueckner [112]. It is worth pointing out here that by contrast with most competing approximate methods, each term generated by $\operatorname{SUB}(2)$ is finite, and no (cancellations between) spurious logarithmic singularities occur. This particular point stems from the more general feature of the CCM 
that terms which tend to cancel each other are automatically grouped together or never split apart.

In the even more interesting strong-coupling limit, $r_{s} \rightarrow \infty$, one may not expect the $\operatorname{SUB}(2)$ approximation based on the condensate model state to be at all reasonable, since one imagines that $n$-body clusters with $n \gg 2$ are now important. Indeed, we expect the system to Wigner crystallize below some critical density, and such a crystal phase is an archetype of a situation where $N$-body correlations dominate. The translational-invariance symmetry of $|\Phi\rangle$ and the Hamiltonian is also spontaneously broken in the crystalline phase. However, in the $\operatorname{SUB}(2)$ approximation we find the behaviour:

$$
\varepsilon \underset{r_{s} \rightarrow \infty}{\longrightarrow}-A r_{s}^{-1}+B r_{s}^{-3 / 2}+O\left(r_{s}^{-2}\right)
$$

and we also see that the KE term contributes first to the constant $B$ in leading order. Although each of the terms RPA, CP and LAD is necessary for a quantitative evaluation of the constant $A$, they play quite different physical roles. Whereas the RPA terms continue to be vital for the correct analytic behaviour by providing the correct long-range $(q \rightarrow 0)$ Coulomb screening, the CP now plays a similarly crucial role in the short-range $(q \rightarrow \infty)$ limit. Although the inclusion of the LAD term quantitatively changes the constants $A$ and $B$, it may safely be omitted without changing the analytic form of Eq. (51) for the low-density energy expansion.

Dropping the LAD term, for example, leads to the analytical results $A=$ $\left(32 / 3 \pi^{2}\right)^{1 / 3} \approx 1.03$ and $B=\sqrt{3} \pi / 8 \approx 0.68$. The virial theorem may also be used to show that in this low-density expansion the leading term is purely potential energy, whereas the much more interesting second term in Eq. (51) has equal kinetic and potential energy contributions. This result is strongly indicative of the simple harmonic motion expected of a (classical) solid. Indeed, Wigner first pointed out that in this limit, where the potential energy dominates, the energy is minimized by the particles crystallizing; and this leads to an electrostatic energy proportional to $r_{s}^{-1}$. Whereas in a fluid phase the particles are free to occupy the whole volume, and hence by the uncertainty principle have a kinetic energy proportional to $r_{s}^{-2}$, the particles in the Wigner solid are constrained to oscillate about fixed lattice sites and hence to have a greater kinetic energy. By simple harmonic motion considerations, this latter energy is easily seen to vary as $r_{s}^{-3 / 2}$.

Thus, our SUB(2) approximant for the ground-state energy has the correct analytic form of a solid, even though the coefficients are not very close to the b.c.c. crystal values of $A \approx 1.792$ and $B \approx 2.65$. (We note that the inclusion of the LAD term only increases this discrepancy by reducing the value of $A$ by about $20 \%$.) What is most important however is that even the lowest $\operatorname{SUB}(2)$ approximation of the CCM gives a low-density energy which cannot possibly represent a fluid phase since the particles are not free to occupy the whole volume. It is clear that the translationally invariant approach based on the condensate model state, well describes both the plasma (fluid) and symmetrybroken solid phases. Although it is at first sight surprising that the fluid-like SUB(2) wavefunction is capable of describing an (amorphous or glassy-state approximation to the crystalline) solid phase, it is apparent that strong manybody correlations are indeed built into the wavefunction. Nevertheless, the coupling terms to $S_{3}$ and $S_{4}$ are still non-negligible, and need to be incorporated for a better quantitative treatment. Conversely, having observed from the 
ground-state CCM energy calculation alone that a transition to a solid-like phase has occurred, one could now do a much better job on such a phase by choosing a new model state $|\Phi\rangle$ that contained the symmetry of some imposed lattice rather than the previous perfect translational invariance.

We turn next to the fermionic Coulomb plasma, and for ease restrict ourselves to the unpolarized spin- $\frac{1}{2}$ "electron gas". The translationally-invariant analogue of Eq. (42) is now the usual filled Fermi sea, $\left|\Phi_{F}\right\rangle$, with a Fermi momentum given by Eq. (39). In the thermodynamic limit the fermionic $S U B(2)$ analogue of $\mathrm{Eq}$. (47) is a nonlinear integral equation in three three-momentum variables for the antisymmetrized matrix elements.

$$
S_{2}^{\sigma_{1} \sigma_{2}}\left(\vec{k}_{1}, \vec{k}_{2} ; \vec{q}\right) \equiv\left\langle\vec{k}_{1}+\vec{q}, \sigma_{1} ; \vec{k}_{2}-\vec{q}, \sigma_{2}\left|S_{2}\right| \vec{k}_{1} \sigma_{1} ; \vec{k}_{2} \sigma_{2}\right\rangle_{A},
$$

which now depend not only on a momentum transfer $\vec{q}$ as for bosons, but also on the two hole-state momenta $\vec{k}_{1}$ and $\vec{k}_{2}$ inside the Fermi sphere, as well as the spin-projection indices $\sigma_{1}, \sigma_{2}\left(= \pm \frac{1}{2}\right)$. The corresponding $S U B(2)$ equation for fermions is therefore algebraically much more complex than Eq. (47). It has been described fully elsewhere [110a].

Once again, it can be shown [110] that in the weak-coupling $\left(r_{s} \rightarrow 0\right)$ plasma regime, the fermionic SUB(2) equation reduces to a leading contribution which is the complete analogue of the KE and RPA terms of Eq. (44). The RPA $[21,22]$ is well known to give exactly the leading high-density contribution to the correlation energy, $\varepsilon_{c}$, where:

$$
\varepsilon \equiv \varepsilon_{0}+\varepsilon_{c} ; \quad\left\langle\Phi_{F}|H| \Phi_{F}\right\rangle \equiv N \varepsilon_{0}\left(\frac{e^{2}}{2 a_{0}}\right) .
$$

The corresponding nonlinear RPA equation for $S_{2}$ has been solved exactly [110a], and the leading (logarithmic) contribution to $\varepsilon_{c}$ at high densities has been verified.

In the intermediate-coupling regime $\left(1 \leqslant r_{s} \leqslant 5\right)$ of metallic densities, we no longer expect RPA to suffice. Quite apart from ignoring (i) the simple exchange terms needed to antisymmetrize RPA, we have neglected even in $\operatorname{SUB}(2)$ approximation: (ii) all combined particle-particle and hole-hole ladder terms, some at least of which are important for correct short-range behaviour; (iii) the self-energy correction terms which self-consistently generate both the particle potential and the (more important) hole potential; (iv) classes of higher ringexchange terms to preserve overall antisymmetry; and (v) other exchange terms which include the particle-hole ladders. Since the full $\operatorname{SUB}(2)$ equation is so technically complicated, a "state-averaging" approximation method was introduced [110] in order to proceed systematically beyond RPA. It was motivated by analogy with the mathematically much simpler Bose equations (44). The basic approximation is to average inside the Fermi sea over the hole momenta $\vec{k}_{1}$ and $\overrightarrow{k_{2}}$ in $S_{2}\left(\vec{k}_{1}, \overrightarrow{k_{2}} ; \vec{q}\right)$, where here and henceforth we drop the spin-projection indices for ease. However in performing this averaging the very important Pauli exclusion principle is preserved by insisting that the particle momenta $\left(\overrightarrow{k_{1}}+\vec{q}\right)$ and $\left(\overrightarrow{k_{2}}-\vec{q}\right)$ simultaneously lie outside the Fermi sea. In this way the exact $S_{2}\left(\vec{k}_{1}, \vec{k}_{2} ; \vec{q}\right)$ is replaced by an approximate state-averaged $\bar{S}_{2}(q)$, and the corresponding CCM equation must itself still be suitably state-averaged.

This latter step is not unique. Nevertheless, this may be put to advantage by bringing physical intuition to bear. Another most important point is that since exact results are known for $S_{2}$ in the RPA limit, any averaging procedure may be checked in at least this case. As a simple illustration we imagine putting this 
scheme into effect for RPA itself. Within the CCM this comprises an equation for $S_{2}$ which involves only the kinetic energy (KE) and RPA terms analogous to the first two terms in the bosonic Eq. (44). After the replacement $S_{2} \rightarrow \bar{S}_{2}$, the only remaining hole-state dependence is in the KE term which has the familiar form:

$$
\frac{1}{2 m}\left[\left|\vec{k}_{1}+\vec{q}\right|^{2}+\left|\vec{k}_{2}-\vec{q}\right|^{2}-k_{1}^{2}-k_{2}^{2}\right] S_{2}\left(\vec{k}_{1}, \vec{k}_{2} ; \vec{q}\right) \equiv e S_{2} .
$$

As two obvious averaging schemes one could imagine either (i) replacing $e \rightarrow\langle e\rangle$; or (ii) the intuitively and physically more appealing replacement $e^{-1} \rightarrow\left\langle e^{-1}\right\rangle$ after first dividing through by $e$; i.e. averaging the two-body propagator or "energy denominator".

Precise details for performing these averages are described in Ref. [110b], where it is also shown that the former procedure, $e \rightarrow\langle e\rangle$, leads precisely to the so-called "mean spherical approximation" discussed by Zabolitzky [113] in this context. This approximation arose in the $r_{s} \rightarrow 0$ limit in his state-independent variational treatment of the electron gas via a Fermi hypernetted chain (FHNC) type of procedure. Whereas this mean spherical approximation is in error for the correlation energy $\varepsilon_{c}$ by $8.4 \%$ in comparison with exact RPA results, it was also shown that the energy denominator averaging $e^{-1} \rightarrow\left\langle e^{-1}\right\rangle$, is exact at $r_{s} \rightarrow 0$, and to give for the RPA better than $2 \%$ accuracy at all densities by comparison with the exact results obtained earlier [110a]. Furthermore, it is argued that there is no reason to expect worse accuracy (and, indeed, several reasons to expect better accuracy) for all remaining terms in the Fermi CCM SUB(2) equation.

This state-averaging procedure was then carried out on the full $\operatorname{SUB}(2)$ equations, and indeed with the additional inclusion of some of the most important contributions from the coupling to $S_{3}$ and $S_{4}$. These included the replacement of the bare Coulomb potential $V$ in $\operatorname{SUB}(2)$ terms other than those directly responsible for generating the two-body ladder diagrams, by a self-consistent $G$-matrix (i.e., one obtained from the full $S_{2}$ solution itself). Such attention to these and other important effects caused by the interference at intermediate separations of the long-range RPA and short-range ladder-sum effects, is necessary for a precise quantitative evaluation of the correlation energy. Results from such calculations were first presented by Bishop and Lührmann [110]. They were later repeated by Emrich and Zabolitzky [114]. These latter authors also avoided the use of several additional unnecessary approximations made by the former, and their CCM results are shown in Table 1. They are labelled CC(4) to indicate that they include at least part of the contributions from triple and quadruple excitations.

Finally, we compare in Table 1 the $\mathrm{CC}$ results with the Green's function Monte Carlo results [115] which come from an essentially exact (apart from statistical errors) stochastic simulation of the many-body Schrödinger equation. We also compare with representative results from the best of the other available calculations, namely the variational calculation of Zabolitzky [113] already alluded to above, and a more phenomenological calculation of Vashishta and Singwi [116]. It is clear that over the entire metallic density regime the CCM results are well within the comparable benchmark of chemical accuracy discussed in Sect. 1. Indeed, we know of no better microscopic description of the electron gas at these densities.

We note that excited states of the one-component Coulomb plasmas may also be investigated within the CCM, although relatively little work has been 
Table 1. Correlation energy per particle (in milli-Rydbergs) of the unpolarized electron gas, for various values of the dimensionless coupling constants, $r_{s}$

\begin{tabular}{lccccccccc}
\hline \multirow{2}{*}{ Method } & \multicolumn{10}{c}{$r_{s}$} \\
\cline { 2 - 11 } & $\rightarrow 0$ & 1 & 2 & 3 & 4 & 5 & 6 & 10 & 20 \\
\hline RPA & $62.2 \ln r_{s}$ & -158 & -124 & -106 & -93.6 & -84.9 & -78.2 & -61.3 & -42.8 \\
CC(4) & $62.2 \ln r_{s}$ & -122 & -90.4 & -73.8 & -63.4 & -56.0 & -50.5 & -37.0 & -23.6 \\
GFMC $^{\mathrm{b}}$ & {$\left[62.2 \ln r_{s}\right]$} & -121 & -90.2 & {$[-73.8]$} & {$[-63.6]$} & -56.3 & {$[-50.7]$} & -37.22 & -23.00 \\
FHNC $^{\mathrm{c}}$ & $57.0 \ln r_{s}$ & -114 & -85.9 & -71.0 & -61.2 & -54.1 & - & -35.5 & -21.8 \\
VS $^{\mathrm{d}}$ & - & -130 & -98 & -81 & -70 & -62 & - & - & - \\
\hline
\end{tabular}

${ }^{a} \mathrm{CCM}$ results of Emrich and Zabolitzky [114]

' Green's function Monte Carlo results of Ceperley and Alder, with the results in brackets obtained by the interpolation procedure of Vosko et al. [115]

${ }^{c}$ Results of a Fermi hypernetted chain type of calculation of Zabolitzky [113]

${ }^{d}$ Results of a phenomenological approach of Vashishta and Singwi [117]

done [110c]. In particular, the well-known "giant resonance" (or collective excitation) for the electron gas at high densities, namely the plasmon mode, may be identified via the $\operatorname{SUB}(1,2)$ approximation. Other authors have also investigated the electron gas using CC techniques (and see, e.g., Ref. [117]). In particular, Arponen and Pajanne have given an ingenious mapping of the fermionic problem onto an equivalent bosonic problem, which is then itself treated by $\mathrm{CC}$ techniques. Finally, we note that the $\mathrm{CC}$ techniques have also been formulated for two-component Fermi systems, using both a "normal" (filled Fermi sea) model state and a "superfluid" (BCS) model state [118].

\section{CC applications to model many-body problems}

We turn next to some applications of $\mathrm{CC}$ techniques that have been made to various model systems in quantum many-body theory. Such systems are especially useful either because they are exactly integrable, and hence provide exact benchmark results against which universal methods may be tested, or because they are simple enough so that such features as the convergence properties of the approximation schemes (e.g., the standard SUB $(n)$ scheme for ground states or the $\operatorname{SUB}(m, n)$ scheme for excited states) may be numerically (or even analytically) examined. Since the specific details of these models are perhaps of less direct interest for quantum chemistry applications, we review them only very briefly. Nevertheless, we stress that such applications are important for the formal development and deeper understanding of the CCM. The specific model systems that we discuss here are: (i) the discrete Lipkin-Meshkov-Glick quasispin model [79]; (ii) the continuous one-dimensional Lieb model [119] of bosons interacting via repulsive delta-function potentials; (iii) one-body quantum anharmonic oscillators considered as model bosonic field theories with a single mode; and (iv) the discrete analogues of the latter in the form of anharmonic spin systems [103c]. 


\subsection{The Lipkin-Meshkov-Glick quasispin model}

The model many-body Hamiltonian of Lipkin, Meshkov, and Glick [79] (the LMG model) was originally invented to simulate the collective monopole vibrations in spherical atomic nuclei. At the most general level, interest in such models as the LMG centres on the fact that they exhibit a phase or shape transition at some critical value of a coupling parameter in the regime for which perturbative techniques are invalid, and for which the RPA would give imaginary values for the excitation energy of a collective mode. Such an occurrence is usually taken as evidence that the unperturbed or model ground state is unstable against some collective vibrational mode, and that another solution of the Hartree-Fock equations exists which exhibits a finite deformation of this particular mode. It is precisely in this context that Kümmel [109] performed his "shape instability analysis" within the CCM, to which we have already referred above. In nuclear physics, the transition from spherical to deformed shape which occurs in the regime of the rare-earth nuclei is believed to be due to a collective quadrupole excitation of this type, and the LMG model was first introduced as its simpler monopole analogue.

The LMG model thus consists of $N$ identical fermions distributed between two energy levels with energy difference $\varepsilon$, each of which is $N$-fold degenerate. The Hamiltonian is taken to be:

$$
H=\frac{1}{2} \varepsilon \sum_{p, m} m a_{p, m}^{\dagger} a_{p, m}+\frac{1}{2} V \sum_{p, p^{\prime}, m} a_{p, m}^{\dagger} a_{p^{\prime}, m}^{\dagger} a_{p^{\prime},-m} a_{p,-m},
$$

where the quantum number $p=1,2, \ldots, N$ labels the degenerate single-particle states within each level, the index $m=-1,+1$ labels the lower and upper levels respectively, and the operators $\left\{a_{p, m}^{\dagger}\right\}$ and $\left\{a_{p, m}\right\}$ respectively create and destroy a fermion in the state $(p, m)$. They obey the usual anticommutation relations:

$$
\left\{a_{p, m}, a_{p^{\prime}, m^{\prime}}^{\dagger}\right\}=\delta_{p p^{\prime}} \delta_{m m^{\prime}} ; \quad\left\{a_{p, m}, a_{p^{\prime}, m^{\prime}}\right\}=0 .
$$

The two-body matrix elements are simply all set to the same constant value $\frac{1}{2} V$, and the Hamiltonian has the form of a simple pairing model in which a pair of particles in the lower (upper) level may be scattered only into the corresponding levels in the upper (lower) level with the same values of the quantum number $p$.

The construction of such models is predicated on the observation that bilinear products of creation and annihilation operators may be considered as generators of Lie groups. In the present case one may define quasispin operators:

$$
\begin{aligned}
& J_{+} \equiv \sum_{p} a_{p,+1}^{\dagger} a_{p,-1} \equiv J_{x}+i J_{y}, \\
& J_{-} \equiv \sum_{p} a_{p,-1}^{\dagger} a_{p,+1} \equiv J_{x}-i J_{y}, \\
& J_{z} \equiv \frac{1}{2} \sum_{p, m} m a_{p, m}^{\dagger} a_{p, m},
\end{aligned}
$$

and hence show from the relations (56) that they obey the usual angular momentum commutation rules of $\mathrm{SU}(2)$ :

$$
\left[J_{+}, J_{-}\right]=2 J_{z}, \quad\left[J_{z}, J_{ \pm}\right]= \pm J_{ \pm} .
$$


The LMG Hamiltonian of Eq. (55) is then trivially rewritten in the exact equivalent quasispin form:

$$
H=\varepsilon\left[J_{z}+\frac{1}{2} g N^{-1}\left(J_{+}^{2}+J_{-}^{2}\right)\right]
$$

where $g \equiv N V / \varepsilon$ is the dimensionless interaction strength parameter. It is easy to see that this Hamiltonian conserves the total quasispin and parity, since it commutes with the corresponding operators:

$$
\begin{aligned}
{\left[H, \vec{J}^{2}\right]=0 ; } & \vec{J}^{2} \equiv J_{x}^{2}+J_{y}^{2}+J_{z}^{2}, \\
{[H, \Pi]=0 ; } & \Pi \equiv \exp \left[i \pi\left(J_{z}+\frac{1}{2} N\right)\right] .
\end{aligned}
$$

An obvious choice of CCM model state is the Slater determinant with all particles in the lower level:

$$
|\Phi\rangle=\prod_{p=1}^{N} a_{p,-1}^{\dagger}|0\rangle,
$$

where $|0\rangle$ is the vacuum state, $a_{p, m}|0\rangle=0$. The state $|\Phi\rangle$ clearly also has the property:

$$
J_{-}|\Phi\rangle=0
$$

Furthermore, it is an eigenstate of the operators $\vec{J}^{2}$ and $J_{z}$ with respective eigenvalues $j(j+1)$ and $-j$, where $j=\frac{1}{2} N$. Due to Eq. (60a) the interacting ground state may be sought from the $(2 j+1)$-dimensional subspace spanned by the vectors $\{|j, m\rangle ; m=-j,-j+1, \ldots, j\}$ where $|j, m\rangle \propto\left(J_{+}\right)^{j+m}|j,-j\rangle$, and we have equivalently to Eq. (61):

$$
|\Phi\rangle=|j,-j\rangle
$$

Physically, the LMG model displays an interesting shape transition in the vicinity of a critical coupling strength $g_{c}$, where $g_{c} \rightarrow 1$ as $N \rightarrow \infty$ [120]. For finite $N$, and working in the RPA (or time-dependent Hartree-Fock approximation), we find that the excitation energy of the collective excitation becomes zero at $g_{c}^{\mathrm{RPA}}=N /(N-1)$. This signals an actual shape transition to a state for $g>g_{c}$ with nonzero value of the order parameter $\left\langle J_{y}\right\rangle$, whereas $\left\langle J_{y}\right\rangle=0$ for $g\left\langle g_{c}\right.$. The symmetric Hartree-Fock solution $|\Phi\rangle$ of Eq. (61) becomes unstable in the RPA for $g>g_{c}^{\text {RPA }}$, and in this region there are two degenerate Hartree-Fock solutions $\left|\Phi_{ \pm}^{\prime}\right\rangle[109]$, which break the parity symmetry:

$$
\begin{gathered}
\left|\Phi_{ \pm}^{\prime}\right\rangle=\prod_{p=1}^{N} b_{p,-1}^{\dagger}|0\rangle \\
b_{p,-1}^{\dagger}=\cos \left(\frac{1}{2} \alpha\right) a_{p,-1}^{\dagger}+i \sin \left(\frac{1}{2} \alpha\right) a_{p,+1}^{\dagger} ; \quad \alpha= \pm \cos ^{-1}\left[\frac{N}{(N-1) g}\right] .
\end{gathered}
$$

Of course, the transition is not actually sharp for finite $N$ since the two broken-symmetry solutions can communicate by quantum tunneling through a finite potential barrier. Thus, for large $N$ and/or large $g$, the lowest states form closely-spaced parity doublets. The true ground state, for example, has even parity, and the first excited state has odd parity and an excitation energy which becomes exponentially small for large $N$ in the region $g>g_{c}$.

The ground-state energy of the LMG was first studied in the NCCM by Lührmann [121], and the excitation spectrum was similarly examined within the NCCM by Emrich [ $81 \mathrm{~b}$ ]. Both of these authors studied only the symmetric 
phase, for values $g \lesssim 0.9$, employing respectively the standard $\operatorname{SUB}(n)$ and $\operatorname{SUB}(m, n)$ schemes. Lührmann in particular showed that the CC results were at least as good as those obtained by such more traditional approaches as the RPA, Brueckner theory, and MBPT. On the other hand, Arponen [122] has shown that not only can the SUB(1) approximation based on the state $|\Phi\rangle$ of Eq. (61) not produce the correct Hartree-Fock deformed states $\left|\Phi_{ \pm}^{\prime}\right\rangle$ of Eq. (64), but nor can the higher NCCM SUB $(n)$ approximations give reasonable results in the deformed phase $g \gtrsim 1$, even when symmetry breaking is introduced by allowing odd-indexed cluster amplitudes (which are otherwise zero for a parity eigenstate). The NCCM gives good results in the deformed region only be employing a more realistic non-spherical model state of the type given in Eq. (64), found by some external means such as that of Ref. [109], and by redefining the creation and annihilation operators with respect to this state. We note that this situation is familiar in many perturbative approaches to quantum field theory, which require the field to be quantized around that particular broken-symmetry model vacuum state which most closely resembles the true ground state.

By contrast, the ECCM has been shown $[74,122]$ to be able to find the appropriate deformed model state completely automatically. The ECCM is capable of spanning the region of the shape transition using a single model state, to give an excellent description of the energy spectrum in both the symmetric and deformed phases $[102,122]$. The sensitivity of the method to the choice of model state has also been examined [102] by using both the symmetric and brokensymmetry Hartree-Fock states, which are themselves fully equivalent to the ECCM SUB(1) solutions, whether or not symmetry breaking occurs.

The overall quality of the ECCM results for the LMG is extremely encouraging, even at relatively low levels of truncation, outside a narrow critical region whose width decreases as $N$ increases. Indeed, we know of no equally fundamental microscopic formalism that can give better overall results for this model. With a single model state one can accurately reproduce within the ECCM virtually all of the quantitative and qualitative details of the energy spectrum. The only noticeable exception is the splitting of the nearly degenerate parity doublets that occur for $g \gg 1$. It seems that a fundamentally new, broken-symmetry (global) version of the multireference $\mathrm{CC}$ approach is needed here, as has been discussed in Ref. [102]. We return to this point in Sect. 10.

\subsection{The one-dimensional exactly integrable Lieb model}

The so-called Lieb model [119] was introduced in 1963. It comprises a gas of $N$ identical bosons constrained to move in a one-dimensional box of length $L$, and interacting via a pairwise repulsive delta-function potential. In units in which $\hbar=2 m=1$, where $m$ is the mass of each boson, the Hamiltonian has the form:

$$
H=-\sum_{i=1}^{N} \frac{d^{2}}{d x_{i}^{2}}+2 c \sum_{i<j=1}^{N} \delta\left(x_{i}-x_{j}\right) ; \quad c>0 .
$$

We are most interested in the thermodynamic limit where $N \rightarrow \infty$ such that $\varrho \equiv N / L$ remains finite. Like the electron gas the Lieb model is again conceptually simplified by the fact that it has only one dimensionless coupling constant, $\gamma \equiv c / \varrho$. The strong-coupling limit $\gamma \rightarrow \infty$ corresponds precisely to the case of one-dimensional hard-core bosons, first solved by Girardeau [123]. 
Lieb and Liniger demonstrated that the Hamiltonian of Eq. (65) is exactly integrable for all values of $\gamma$. By considering a Bethe-ansatz [124] type of wavefunction they could solve for both the ground state [119a] and the excited states [119b], and in the thermodynamic limit they derived integral equations for the corresponding energies. The system has two interesting features. In the first place, the elementary excitation spectrum for all $\gamma>0$ looks like that of ideal fermions (rather than of ideal bosons). The second point is more subtle, but it seems to be a general feature of exactly soluble models. Thus, the solution to the integral equation for the ground-state energy is simple to obtain in the strongcoupling regime, $\gamma \rightarrow \infty$, whereas as $\gamma \rightarrow 0$ the solution becomes singular. By contrast, the simple Bogoliubov approximation becomes asymptotically exact in this latter limit although, as Lieb has pointed out, this approximation is itself very difficult to justify rigorously. Finally, we also note that the Lieb model is the continuum limit of the one-dimensional discrete chain model for spin- $\frac{1}{2}$ particles (with spins $\vec{s}_{i}$ on the sites $i$ of the chain) interacting via the nearest-neighbour XXZ-model (or anisotropic Heisenberg model) Hamiltonian:

$$
H=\sum_{i=1}^{N}\left(s_{i}^{x} s_{i+1}^{x}+s_{i}^{y} s_{i+1}^{y}+\Delta s_{i}^{z} s_{i+1}^{z}\right),
$$

which is also discussed in Sect. 8 .

In many ways the electron gas (Coulomb interaction) and the Lieb model (delta-function interaction) are archetypes of systems dominated respectively by long- and short-range interactions. It is therefore also of interest to apply our universal many-body techniques to the Lieb model. A very limited amount of work has been done in this regard [78, 125]. We find that both the NCCM and ECCM give good results at low truncation levels in the weak-coupling regime, $\gamma \rightarrow 0$, using for the model state $|\Phi\rangle$ either the number-conserving condensate of Eq. (42) or the number-nonconserving field-theoretic vacuum. However, neither method in the standard $\operatorname{SUB}(n)$ truncation scheme gives the correct analytic behaviour of the ground-state energy for practicable values of the index $n$ in the strong-coupling regime, $\gamma \rightarrow \infty$. By contrast, parquet theory and Jastrow variational techniques seem to perform much better. This has led us to suggest new CCM truncation schemes [78], based on a detailed study of the similarities and differences between the two methods. We elaborate on this point in Sect. 10.

\subsection{Anharmonic oscillators; single-mode bosonic field theories}

The one-dimensional, single-well quartic anharmonic oscillator is described by the one-body Hamiltonian:

$$
H=\frac{1}{2} p^{2}+\frac{1}{2} x^{2}+\lambda x^{4} ; \quad \lambda>0,
$$

(in units where $\hbar=m=1$, and $m$ is the particle mass), where $p \rightarrow-i d / d x$. It is an important system in quantum mechanics because it is known that RayleighSchrödinger perturbation theory about the $\lambda=0$ unperturbed harmonic oscillator fails to converge for all values of $\lambda$, however small [126]. Furthermore, by introducing the usual destruction operator $a \equiv 2^{-1 / 2}(x+i p)$ and its Hermitian adjoint $a^{\dagger}$, Eq. (67) may be written in the equivalent form:

$$
H=\frac{1}{2}+a^{\dagger} a+\frac{1}{4} \lambda\left(a+a^{\dagger}\right)^{4},
$$


namely as a single-mode bosonic field theory, since $a$ and $a^{\dagger}$ obey the usual bosonic commutation relation:

$$
\left[a, a^{\dagger}\right]=1 \text {. }
$$

Hamiltonians of the form of Eq. (68) are just the $(0+1)$-dimensional analogues of real $(d+1)$-dimensional field theories in a nonzero number $d$ of space dimensions and one time dimension. Considered as just such a model field theory, the anharmonic oscillator also provides an extremely stringent test of many-body formulations like the CCM which are specifically designed for local field theories or systems with normal locality and separability properties. By contrast, the Hamiltonian of Eq. (68) is highly singular in the sense of exhibiting maximal nonlocality, and possessing no attribute of separability or size-extensivity.

In order to apply CC techniques to Eq. (68), an obvious choice for the model state $|\Phi\rangle$ is the vacuum state $\left|0_{a}\right\rangle$ for the bosons of Eq. (69), $a\left|0_{a}\right\rangle=0$. On the other hand, one may also consider making a (linear, canonical) Bogoliubov transformation $[127,128]$ to a new bosonic basis:

$$
\begin{gathered}
b \equiv\left(1-t^{2}\right)^{-1 / 2}\left(a-t a^{\dagger}\right) ; \quad|t|<1, \\
{\left[b, b^{\dagger}\right]=1,}
\end{gathered}
$$

and similarly to employ the vacuum state $\left|0_{b}\right\rangle$, where $b\left|0_{b}\right\rangle=0$, as model state $|\Phi\rangle$. In the former case the Fock space is spanned by a complete, orthonormal set of creation operators $\left\{C_{I}^{\dagger}\right\} \rightarrow\left\{(n !)^{-1 / 2}\left(a^{\dagger}\right)^{n} ; n=0,1,2, \ldots\right\}$, and similarly in the latter case by $a^{\dagger} \rightarrow b^{\dagger}$. The free parameter $t$ may be chosen by one of several (variational) criteria. For example, the Hartree choice minimizes the expectation value $\left\langle 0_{b}|H| 0_{b}\right\rangle$; whereas the maximum overlap condition maximizes the quantity $\left\langle 0_{b} \mid \Psi\right\rangle$ where $|\Psi\rangle$ is the (normalized) exact ground state. One expects that some such "good" choice of the parameter $t$ will accelerate the convergence of subsequent truncation schemes.

The CI method, NCCM, and ECCM may now all be put into effect in their respective standard $\operatorname{SUB}(n)$ and $\operatorname{SUB}(m, n)$ truncation schemes for ground and excited states. One of the great advantages of the simplicity of the model is that one may proceed computationally to very high values of the truncation indices $m$ and $n$ in order to investigate the convergence properties of the individual methods numerically. Several such investigations have now been performed $[82,103,127-131]$, the most ambitious of which use truncation indices $m, n \lesssim 30$. Recent investigations $[103,104]$ have also explored the structure of the exact NCCM and ECCM representations much more deeply, by employing the holomorphic or Bargmann-space representation in which we replace $a \rightarrow d$ I $d z, a^{\dagger} \rightarrow z$, in terms of a complex number $z$.

For relatively low values of the truncation indices $m$ and $n$ in the standard $\operatorname{SUB}(m, n)$ scheme based on the Hartree vacuum as model state, for example, both the NCCM and ECCM give very good values for the energy levels of this very demanding model. For example, for the ground-state energy, the $\operatorname{SUB}(6)$ truncations give values which are accurate to better than $0.1 \%$ over the entire range of $\lambda$. Nevertheless, for much higher values of $n$ in the NCCM, this very rapid initial convergence slows markedly and shows signs of the oscillatory behaviour characteristic of asymptotic series [129-131]. Similar calculations (i.e., with $n \lesssim 30$ ) have also been performed within the ECCM [103c], but the computational difficulties here are even more pronounced. It is probably fair to say that for neither the NCCM nor the ECCM are these numerical studies 
conclusive. Nevertheless, they support the idea that both methods show rapid initial convergence (which in practice is all that is required for more realistic systems), followed by the series eventually becoming at best asymptotic. We note that similar calculations have also been performed for the much more technically demanding asymmetric (cubic-plus-quartic) anharmonic single-well and the symmetric quartic double-well potentials [131].

Finally, we note that the Bargmann-space methods [103] have already provided us with considerable insight into the structure of the NCCM and ECCM parametrizations. Since they are the subject of a separate article in the present volume [103e], we give no detailed discussion here. We stress only that the holomorphic representation provides a complete algebraization of the otherwise abstract, topological linking and double-linking structures associated with the vertices of the emergent tree diagrams that arise in the NCCM and ECCM, respectively. Furthermore, it gives a definite mechanism to regularize the formally divergent series that appear in both $\mathrm{CC}$ representations when otherwise convergent, exact integral expressions are (incorrectly) expanded.

\subsection{Anharmonic spin models}

Many of the problems associated with the various divergent series that arise in the expressions for the cluster coefficients which characterize the NCCM and ECCM parametrizations of the above anharmonic oscillator, stem from the fact that its Hilbert space is infinite-dimensional. One might hope that another way of shedding light on these problems is to examine a finite-dimensional analogue from which it smoothly evolves in some appropriate limit. One way of doing this is to consider the $(2 j+1)$-dimensional spin space corresponding to a spin of quantum number $j$. In terms of the original SU(2) operators $\left(J_{ \pm}, J_{z}\right)$ which obey the usual spin commutation relations of Eq. (58), we define a set of new operators:

$$
\mathscr{J}_{0} \equiv J_{z}+j ; \quad \mathscr{J}_{ \pm}=(2 j)^{-1 / 2} J_{ \pm}
$$

It is then easy to check that for large values of $j$ the commutation relations between the operators $\mathscr{J}_{+}, \mathscr{J}_{-}$, and $\mathscr{J}_{0}$ are the same as those between the bosonic operators $a^{\dagger}, a$, and $a^{\dagger} a$, respectively, except for terms of order $O\left(\left\langle a^{\dagger} a\right\rangle \mid j\right)$. Hence, in the limit that $j \rightarrow \infty$, the anharmonic spin Hamiltonian, $H_{j}$, defined as:

$$
H_{j}=\frac{1}{2}+j+J_{z}+\frac{\lambda}{16 j^{2}}\left(J_{+}+J_{-}\right)^{4},
$$

should smoothly approach the anharmonic oscillator Hamiltonian of Eq. (68), in the well-defined sense that any energy eigenvalue $E_{n}^{(j)}$ with fixed value of $n$ should converge to the corresponding anharmonic oscillator eigenvalue $E_{n}$.

The discrete spin-Hamiltonian of Eq. (72) may now be exactly diagonalized by ordinary matrix techniques for any finite value of $j$. In this way the exact values of the corresponding complete sets of NCCM and ECCM amplitudes $\left\{s_{n}, \tilde{s}_{n}\right\}$ and $\left\{\sigma_{n}, \tilde{\sigma}_{n}\right\}$ respectively, where only even values of $n \leqslant 2 j$ enter, may be computed for any value of $\lambda$. Results have been given [103c] for $j \leqslant 60$. We note only that whereas the NCCM amplitudes $\left\{\tilde{s}_{n}\right\}$ converge only very slowly to their $j \rightarrow \infty$ limits, the convergence of the original ECCM amplitudes $\left\{s_{n}, \tilde{\sigma}_{n}\right\}$ is much more rapid, which presumably indicates that this latter set of parameters 
provides a rather stable set. Conversely, the ECCM amplitudes $\left\{\sigma_{n}\right\}$ which are canonically conjugate to the set $\left\{\tilde{\sigma}_{n}\right\}$ do not easily stabilize, and indeed no reliable way of calculating them accurately for larger values of $n$ for these anharmonic spin and oscillator systems currently exists to the best of our knowledge. The interested reader is referred to Ref. [103c] for further details.

\section{CC applications in quantum field theory}

We now turn our attention to the application of $\mathrm{CC}$ techniques to problems in quantum field theory. The group of Kümmel and his coworkers at Bochum has been responsible for most of the developments in this area, and we consider two specific examples.

\section{1. $\Phi^{4}$ field theory}

The basic ideas described in Sect. 6 for the application of the CCM to anharmonic oscillators (and other one-body problems in quantum mechanics) considered as model field theories in $(0+1)$ dimensions, may now quite readily be generalized to the corresponding bosonic field theories in $(d+1)$ dimensions. A prototype which has been much studied in the past by NCCM techniques is $(1+1)$-dimensional $\Phi^{4}$ field theory $[128,132]$. For this model there are two forms of the Hamiltonian density $\mathscr{H}$, namely the so-called symmetric $\left(\mathscr{H}_{s}\right)$ and broken-symmetry $\left(\mathscr{H}_{b}\right)$ forms. Their analogues in the $(0+1)$-dimensional case are the single-well and double-well quartic oscillators. In both cases the theory is defined in a one-dimensional box of length $L$ with Hamiltonian $H$ given as:

$$
H=\int_{0}^{L} d x \mathscr{H}(x) .
$$

The two respective Hamiltonian densities are given analytically as:

$$
\begin{aligned}
& \mathscr{H}_{s}=\mathscr{N}_{m}\left\{\frac{1}{2} \Pi^{2}+\frac{1}{2}(d \Phi / d x)^{2}+\frac{1}{2} m^{2} \Phi^{2}+\frac{1}{4} \lambda \Phi^{4}\right\}, \\
& \mathscr{H}_{b}=\mathscr{N}_{M}\left\{\frac{1}{2} \Pi^{2}+\frac{1}{2}(d \Phi / d x)^{2}-\frac{1}{4} M^{2} \Phi^{2}+\frac{1}{4} \lambda \Phi^{4}\right\},
\end{aligned}
$$

in terms of the field $\Phi=\Phi(x, t)$ and its conjugate momentum density $\Pi=\Pi(x, t)$. These latter may be expanded, as usual, in terms of a complete set of bosonic modes with corresponding operators $\left\{a_{k}, a_{k}^{\dagger}\right\}$ as:

$$
\begin{aligned}
& \Phi(x, t)=\sum_{k}\left(2 \omega_{k} L\right)^{-1 / 2}\left\{a_{k}(t) e^{i k x}+a_{k}^{\dagger}(t) e^{-i k x}\right\} . \\
& \Pi(x, t)=-i \sum_{k}\left(\omega_{k} / 2 L\right)^{1 / 2}\left\{a_{k}(t) e^{i k x}-a_{k}^{\dagger}(t) e^{-i k x}\right\} .
\end{aligned}
$$

The normal-ordering operators $\mathscr{N}_{m}$ and $\mathscr{N}_{M}$ in Eqs. (74a,b) are defined with respect to the operators $\left\{a_{k}, a_{k}^{\ddagger}\right\}$ with the corresponding frequencies $\omega_{k}=\left(k^{2}+m^{2}\right)^{1 / 2}$ and $\omega_{k}=\left(k^{2}+M^{2}\right)^{1 / 2}$, respectively. We note that classically the symmetric $\mathscr{H}_{s}$ has an energy minimum at $\Phi=0$ and oscillator frequency $\omega_{k}=\left(k^{2}+m^{2}\right)^{1 / 2}$, whereas the broken-symmetry $\mathscr{H}_{b}$ is so-called because it has two classical energy minima at $\Phi= \pm M /(2 \lambda)^{1 / 2}$, with an excitation spectrum $\omega_{k}=\left(k^{2}+M^{2}\right)^{1 / 2}$ in both. Once again, it is possible to make a Bogoliubov 
transformation to a new complete set of canonical boson modes $\left\{b_{\alpha}, b_{\alpha}^{\dagger}\right\}$ in complete analogy with Eq. (70) for the single-mode case.

The $(1+1)$-dimensional $\Phi^{4}$ Hamiltonians of Eqs. (73) - (74) are especially interesting in the light of several known exact results, namely:

(i) The energy is a continuous function of the dimensionless coupling constant $g$ (where $g^{s} \equiv \lambda / m^{2}$ and $g^{b} \equiv \lambda / M^{2}$ );

(ii) There is exactly one phase transition, which must therefore be of second order. By Lorentz invariance the excitation energy is of the form $\varepsilon_{k}=\left(k^{2}+\tilde{m}^{2}\right)^{1 / 2}$, and at the critical point $g_{c}$ the energy gap $\tilde{m} \rightarrow 0$. Hence, the two-point Green's function $G\left(x, x^{\prime}\right)$ shows a changeover at the critical point from exponential long-range decay proportional to $\exp \left[-\tilde{m}\left(x-x^{\prime}\right)\right]$, to algebraic decay;

(iii) For $g^{s}>g_{0}^{s} \approx 9.046$ there are two Hamiltonian densities, $\mathscr{H}_{b}^{1}$ and $\mathscr{H}_{b}^{2}$, dual to a given $\mathscr{H}_{s}$, in the sense that all three are equivalent up to additive constants which scale like $L$. For $g^{s}=g_{0}^{s}, \mathscr{H}_{b}^{1} \equiv \mathscr{H}_{b}^{2}$ with a value $g_{0}^{b}=2 \pi / 3$, and for $g^{s}<g_{0}^{s}$ there is no $\mathscr{H}_{b}$ equivalent to a given $\mathscr{H}_{s}$;

(iv) The so-called topological charge $Q$, defined as:

$$
Q=\left(\frac{1}{2} g^{b}\right)^{1 / 2} \int_{0}^{L} d x(d \Phi / d x),
$$

commutes with $H$, and has the eigenvalues $0, \pm 1$. These correspond respectively to the so-called "vacuum sector" and the "soliton (kink and antikink) sectors". The latter occur only for $g^{s}>g_{0}^{s}$.

For details of the CCM results, the reader is guided to the literature $[128,132]$. We note only that in both the vacuum and soliton sectors most exact features of the model can be reproduced, with the exception of the actual critical point. By contrast, this is not the case in the Gaussian (or, roughly speaking, Hartree) approximation. In practice, what occurs in the CCM calculations for the vacuum sector, for example, is that $\mathscr{H}_{b}$ can be studied for all values of $g^{b}$. On the other hand, for $\mathscr{H}_{s}$ there is a region $g^{s}<g_{-}^{s}$ for which the vacuum field expectation value $\langle\Phi\rangle=0$; a region $g^{s}>g_{+}^{s}$ for which $\langle\Phi\rangle \neq 0$; and a region in between, $g_{-}^{s}<g^{s}<g_{+}^{s}$, for which no CCM solution can be found. The width of this region depends on the choice of model state and on the particular approximation scheme used. The region is presumed to contain the actual critical point, $g_{c}$, for lower values of which the symmetric model possesses a unique physical vacuum state, and for higher values of which its vacuum state is spontaneously broken dynamically (by "radiative corrections").

\subsection{A model field theory of pions and nucleons}

Another very interesting problem from the standpoint of quantum chemistry is the standard $(3+1)$-dimensional model of pions and nucleons interacting via the isospin-invariant pseudoscalar coupling. It has a Hamiltonian density given as:

$$
\begin{aligned}
\mathscr{H} & =\mathscr{H}_{0}+\mathscr{H}_{\mathrm{int}}, \\
\mathscr{H}_{0} & =\mathscr{H}_{0}(\vec{x})=\frac{1}{2}\left[\Pi_{t}^{\dagger} \Pi_{t}+\vec{\nabla} \Phi_{t}^{\dagger} \cdot \vec{\nabla} \Phi_{t}+m^{2} \Phi_{t}^{\dagger} \Phi_{t}\right]+\bar{\Psi}_{t^{\prime}}(-i \vec{\gamma} \cdot \vec{\nabla}+M) \Psi_{t^{\prime}}, \\
\mathscr{H}_{\mathrm{int}}(\vec{x}) & =-i g \int d^{3} x^{\prime} F\left(\vec{x}-\vec{x}^{\prime}\right) \bar{\Psi}_{t^{\prime}}(\vec{x}) \gamma_{5} \tau_{t} \Psi_{t^{\prime}}(\vec{x}) \Phi_{t}\left(\vec{x}^{\prime}\right),
\end{aligned}
$$


where $\Phi_{t}=\Phi_{t}(\vec{x})$ and $\Pi_{t}=\Pi_{t}(\vec{x})$ are respectively the pion (bosonic) field operator and its conjugate momentum density operator, as in Eq. (74a), and where $\Psi_{t^{\prime}}(\vec{x})$ and $\bar{\Psi}_{t^{\prime}}(\vec{x})$ are the four-component Dirac nucleon (fermionic) field operator and its adjoint respectively. The matrices $\hat{\gamma}$ and $\gamma_{5}$ are the usual $(4 \times 4)$ Dirac matrices; the three matrices $\tau_{t}$ are the usual $(2 \times 2)$ Pauli isospin matrices; and the summation convention is implied over the repeated isospin indices $t^{\prime}$ for the (isospin- $\frac{1}{2}$ ) nucleon and $t$ for the (isospin-1) pion. The form-factor $F(\vec{x})$ is necessary to renormalize the pion-nucleon vertex. It is taken to have the standard form whose Fourier transform in momentum space is given as:

$$
F(\vec{q})=\frac{\lambda^{2}-m^{2}}{\lambda^{2}+q^{2}},
$$

where $\lambda$ is a high-momentum (or small-distance) cutoff parameter. Finally, the mass parameters $m$ and $M$ are the bare pion and nucleon masses in the theory.

A CCM calculation for this system has been carried out [133] in the multireference ("open-shell") formulation described earlier. In the first place, the physical vacuum $|\Psi\rangle$ is written in terms of the bare vacuum $|\Phi\rangle$ exactly as in Eq. (10), in terms of a correlation operator $S$ which is now expanded as a double sum:

$$
S=\sum_{m, n=1}^{\infty} S_{m n},
$$

in terms of the number $m$ of pions and the number $n$ of nucleon-antinucleon pairs. Secondly, the (physical) one-nucleon state is treated exactly as in the one-valence parametrization of Eq. (19); and, thirdly, the two-nucleon state is treated as in the two-valence parametrization of Eq. (23). The operators $F^{(1)}$ and $F^{(2)}$ are also decomposed as in Eq. (79).

Hasberg and Kümmel [133] have performed such a multireference CCM calculation in which they kept the partitions $S_{01}, S_{11}, F_{10}^{(1)}$ (and $F_{20}^{(1)}, F_{01}^{(1)}$, and $F_{11}^{(1)}$ in low order). Clearly, the results depend on the parameters $M, g$, and $\lambda$. In principle they also depend on the bare pion mass $m$, but the pion self-energy is actually a higher-order effect than the above approximations used, and hence $m$ is set to the physical (experimental) pion mass. The one-nucleon calculation was then used to fit the bare mass $M$ from the $\mathrm{CC}$ prediction for the physical (experimental) nucleon mass. Hence, the two remaining parameters $\lambda$ and $g$ could be used to examine the two-nucleon (deuteron) binding energy. The interested reader is referred to Ref. [133] for further details. We note only that the dependence on $\lambda$ was, very satisfyingly, found to be weak, and that with values of the pion-nucleon coupling constant $g^{2}$ close to the physical ("experimental") value, very good agreement could be found with the experimental deuteron binding energy. The convergence obtained in these pioneering calculations is most impressive, and it indicates yet again that $\mathrm{CC}$ techniques which are by now quite standard in quantum chemistry can find very valuable applications in very diverse areas of physics.

\section{CC applications to quantum spin chain and lattice models}

Quantum spin lattice models are interesting for a number of reasons. From the theoretical viewpoint, they are among the most quantum-mechanical of all known systems, in the sense that many of their properties differ profoundly from 
those of their classical counterparts, in a way which is often very counterintuitive. In particular, they display a fascinating range of phase transitions and associated changes of order and symmetry. Their behaviour can also be studied for various values of the spin quantum number, the coupling constants in the Hamiltonian, and the dimensionality and underlying symmetry properties of the lattice on which they are defined. Simple changes in each of these parameters can lead to profound changes in the nature of the system. Finally, the various quantum spin lattice models also exhibit a diverse mix of exactly integrable and nonintegrable systems.

Experimentally, spin lattice systems are also of great interest, not only for such obvious examples as solid ${ }^{3} \mathrm{He}$, but also for the various magnetic systems displaying ferromagnetism and antiferromagnetism. In this latter regard there exist several quasi-one-dimensional and quasi-two-dimensional systems whose distinctive properties are believed to arise from their strongly anisotropic bonding. In a typical quasi-one-dimensional antiferromagnet, for example, the material may form chains due to a strong superexchange coupling between its constituent magnetic ions (e.g., $\mathrm{Ni}^{2+}$ ). Such materials exist for which the ratio of interchain to intrachain couplings is less than $0.1 \%$. Similarly, many important quasi-two-dimensional layered compounds exist. A particularly topical example is provided by the ceramic copper oxide materials displaying high-temperature superconductivity. Indeed, the two-dimensional (2-d) spin- $\frac{1}{2}$ system defined on a square lattice, with nearest-neighbour antiferromagnetic Heisenberg interactions between the spins, has been hypothesized [134] as a model of relevance for the observed superconductivity, where the belief is that this interaction models that between the electrons in the singly-occuped $d_{x^{2}-y^{2}}$ orbitals on the copper atoms.

Thus, for both strong theoretical and experimental reasons, it is of considerable interest to bring our universal tools of quantum many-body theory to bear upon spin-lattice systems. To date, we are aware of only two such attempts to utilize CCM techniques $[135,136]$. In the first of these, Roger and Hetherington [135] used only the ground-state CCM, and hence restricted themselves to ground-state energies. They were primarily interested in the solid phases of ${ }^{3} \mathrm{He}$, which have proven to be very difficult cases to study theoretically at the microscopic level. By using $\mathrm{CC}$ techniques they have calculated the transition field between the two ordered phases of body-centred cubic (bcc) ${ }^{3} \mathrm{He}$, and they have predicted its variation with some respect to molar volume. Furthermore, they have also predicted some interesting properties of the magnetization of a 2-d square lattice of ${ }^{3} \mathrm{He}$ adsorbed onto some appropriate substrate.

The present author and his coworkers [136] have been more interested in such models as the XXZ-model (or anisotropic Heisenberg) Hamiltonian. The spin $-\frac{1}{2}$ one-dimensional (1-d) chain version of this model, whose Hamiltonian is given in Eq. (66), is exactly soluble by Bethe-ansatz techniques [124], and hence provides a useful benchmark. It displays an interesting phase transition at $\Delta=1$ between an Ising-like antiferromagnetically ordered phase at $\Delta>1$ to a "critical" antiferromagnetic phase for $|\Delta|<1$. The $\Delta>1$ phase exhibits both a gap in its excitation spectrum and a characteristic exponential decay in the spin-spin correlation function to a nonzero value (namely, the order parameter), at large separations of the two spins. By contrast, the critical phase in the region $|\Delta|<1$ has a gapless excitation spectrum, and a spin-spin correlation function that decays algebraically to zero at large separations.

By starting with a perfectly ordered, alternating Néel state on the 1-d chain as our model state $|\Phi\rangle$, we have demonstrated not only that the NCCM can give 
good results for the ground-state and excitation energies, but also that it gives a qualitatively correct description of the entire Ising-like phase as a function of the anisotropy parameter $\Delta$, right down to a predicted critical point $\Delta_{c}$ (which itself depends on the particular approximation). Furthermore, together with the standard SUB $(n)$ scheme, several other completely new truncation schemes have been invented and investigated in this work. We believe that these may potentially be of interest in other fields also, including quantum chemistry. This topic is considered in detail in another article in the present volume [136], to which the interested reader is therefore referred. We note only that similar techniques are now also being applied to the analogous spin- $-\frac{1}{2} \mathrm{XXZ}$ model on a 2-d square lattice, which is nonintegrable and of interest in high-temperature superconductivity. Similar extensions are also in progress for comparable (nonintegrable) spin-1 models. Of special interest here is the microscopic investigation of the very interesting so-called Haldene phase [137], which has been conjectured to exist for the 1-d spin-1 chain and which has no counterpart in the integrable spin $-\frac{1}{2}$ chain.

\section{Other formal applications of the ECCM}

Finally, we turn our attention to two specific applications of the ECCM which have shown how the basic formalism may fairly easily be integrated with rather important gauge-field techniques. The two very different, specific problems concerned are: (i) the (zero-temperature) quantum fluid dynamics of a stronglyinteracting condensed Bose fluid [100]; and (ii) a charged impurity in a polarizable medium [101]. In the former case the gauge fields are external, and correspond to the scalar and vector potentials (arising from the $U(1)$ symmetry of number conservation, and hence exactly as met in electromagnetism) which serve to set the system into its most general state of motion. In the case of the charged impurity, the gauge fields are internal and correspond to the polarization degrees of freedom.

To date, the extensions to deal with both problems have been formulated at a rather general level. Although the theoretical formalism is complete in both cases, practical implementations have not yet been made. Nevertheless, we note that we regard these two successful marriages of gauge-field ideas with $\mathrm{CC}$ methodology as being potentially very important. Thus, for example, nearly all relativistic field theories of interest in particle physics are (usually non-Abelian) gauge field theories. Quantum chromodynamics (QCD) is an obvious example. The above two examples from the nonrelativistic domain thus lend credence to the hope that CC (and particularly ECCM) techniques might be useful in studying such field theories and such particular aspects as the problem of quark confinement.

\subsection{Quantum fluid dynamics of a condensed Bose fluid}

As we have seen in Sect. 3, the ECCM at its most general provides the equations of motion for a complete set of linked-cluster amplitudes which fully characterize the system at hand. It formally decomposes an arbitrary quantum many-body problem based on a Schrödinger dynamics into a nonlocal classical field theory for a set of interacting, $n$-body, $c$-number fields or amplitudes $\left\{\sigma_{n}, \tilde{\sigma}_{n}\right\}$. In coordinate space these are simply $n$-point functions of spatial arguments 
$\left(\vec{x}_{1}, \ldots, \vec{x}_{n}\right)$ and time $t$. For the condensed Bose fluid case in which the model state $|\Phi\rangle$ is now chosen to be the bare vacuum state, it is easy to check that the lowest-order (one-body) amplitudes are precisely the usual condensate wavefunctions, namely the (time-dependent) expectation values of the one-boson creation and destruction operators (or field operators), $a^{\dagger}(\vec{x})$ and $a(\vec{x})$ respectively, for a particle at space-point $\vec{x}$ :

$$
\tilde{\sigma}_{1}(\dot{x} ; t)=\left\langle a^{\dagger}(\vec{x})\right\rangle \equiv\left\langle\tilde{\Psi}(t)\left|a^{\dagger}(\vec{x})\right| \Psi(t)\right\rangle ; \quad \sigma_{1}(\vec{x} ; t)=\langle a(\vec{x})\rangle .
$$

However, the higher-order ECCM amplitudes may also be regarded as generalized quasilocal order parameters. For example:

$$
\tilde{\sigma}_{2}(\vec{x}, \vec{y} ; t)=\left\langle a^{\dagger}(\vec{x}) a^{\dagger}(\vec{y})\right\rangle_{\text {conn }} \equiv\left\langle a^{\dagger}(\vec{x}) a^{\dagger}(\vec{y})\right\rangle-\left\langle a^{\dagger}(\vec{x})\right\rangle\left\langle a^{\dagger}(\vec{y})\right\rangle .
$$

In order to set the system into arbitrary motion, we introduce two external gauge fields $\phi(\vec{x}, t)$ and $\vec{A}(\vec{x}, t)$ which couple respectively to the density operator $\varrho(\vec{r})$ and the current operator $\vec{j}(\vec{r})$ :

$$
\begin{aligned}
& \varrho(\vec{r})=\varrho(\vec{r}, \vec{r}) ; \quad \varrho\left(\vec{r}, \vec{r}^{\prime}\right)=a^{\dagger}(\vec{r}) a\left(\vec{r}^{\prime}\right), \\
& \vec{j}(\vec{r})=\lim _{\vec{r}^{\prime} \rightarrow \dot{r}} \frac{i}{2 m}\left(\vec{\nabla}_{\vec{r}}-\vec{\nabla}_{\vec{r}^{\prime}}\right) \varrho\left(\vec{r}, \vec{r}^{\prime}\right) .
\end{aligned}
$$

For a system of identical bosons of mass $m$ each, we are thus led to consider the grand-canonical Hamiltonian $H$ :

$$
\begin{aligned}
H= & H_{0}+\int d \vec{r}\left[\left\{\phi(\vec{r}, t)+\frac{1}{2 m} \vec{A}^{2}(\vec{r}, t)\right\} \varrho(\vec{r})-\vec{A}(\vec{r}, t) \cdot \vec{j}(\vec{r})\right], \\
H_{0}= & \frac{1}{2 m} \int d \vec{r}\left[\vec{\nabla} a^{\dagger}(\vec{r})\right] \cdot[\vec{\nabla} a(\vec{r})]+\frac{1}{2} \int d \vec{r} \int d \vec{r}^{\prime} V\left(\vec{r}-\vec{r}^{\prime}\right) a^{\dagger}(\vec{r}) a^{\dagger}\left(\vec{r}^{\prime}\right) a\left(\vec{r}^{\prime}\right) a(\vec{r}) \\
& -\mu \int d \vec{r} a^{\dagger}(\vec{r}) a(\vec{r}),
\end{aligned}
$$

where $\mu$ is the chemical potential, introduced since our ECCM states based on the vacuum $|\Phi\rangle$ do not conserve particle number .

Under the effect of a local gauge transformation:

$$
\phi \rightarrow \phi^{\prime}=\phi+\partial \Lambda / \partial t ; \quad \vec{A} \rightarrow \vec{A}^{\prime}=\vec{A}-\vec{\nabla} \Lambda,
$$

with arbitrary phase field $\Lambda=\Lambda(\vec{r}, t)$, the ECCM fields are easily seen to acquire a simple phase factor:

$$
\begin{aligned}
& \sigma_{n}\left(\vec{x}_{1} \ldots \vec{x}_{n} ; t\right) \rightarrow \sigma_{n}^{\prime}\left(\vec{x}_{1} \ldots \vec{x}_{n} ; t\right)=\exp \left[-i \sum_{k=1}^{n} \Lambda\left(\vec{r}_{k}, t\right)\right] \sigma_{n}\left(\vec{x}_{1} \ldots \vec{x}_{n} ; t\right), \\
& \tilde{\sigma}_{n}\left(\vec{x}_{1} \ldots \vec{x}_{n} ; t\right) \rightarrow \tilde{\sigma}_{n}^{\prime}\left(\vec{x}_{1} \ldots \vec{x}_{n} ; t\right)=\exp \left[+i \sum_{k=1}^{n} \Lambda\left(\vec{r}_{k}, t\right)\right] \tilde{\sigma}_{n}\left(\vec{x}_{1} \ldots \vec{x}_{n} ; t\right) .
\end{aligned}
$$

The ECCM equations of motion for the fields $\left\{\sigma_{n}, \tilde{\sigma}_{n}\right\}$, with the grand-canonical Hamiltonian of Eq. (83), may now be derived. For example, their ensuing SUB(1) approximation in which only $\left\{\sigma_{1}, \tilde{\sigma}_{1}\right\}$ are retained, is precisely the (time-dependent mean-field or Hartree or) Gross-Pitaevskii approximation [138], which yields the well-known kink-soliton solution in one-dimensional geometry and the comparable vortex solution in cylindrical geometry. 
Similarly, we may examine the equation of motion of the one-body density matrix:

$$
\bar{\varrho}\left(\vec{r}, \vec{r}^{\prime} ; t\right) \equiv\left\langle\tilde{\Psi}(t)\left|\varrho\left(\vec{r}, \vec{r}^{\prime}\right)\right| \Psi(t)\right\rangle .
$$

By making use of the ECCM parametrizations for $|\Psi(t)\rangle$ and $\langle\tilde{\Psi}(t)|$ from Sect. 3 , it is easy to derive the relation:

$$
\begin{aligned}
\bar{\varrho}\left(\vec{r}, \vec{r}^{\prime} ; t\right) & =\left\langle\Phi\left|\tilde{\Sigma} a^{\dagger}\left(\vec{r}^{\prime}\right) a\left(\vec{r}^{\prime}\right) \Sigma\right| \Phi\right\rangle \\
& =\sum_{n=0}^{\infty} \frac{1}{n !} \int d \vec{x}_{1} \ldots \int d \vec{x}_{n} \tilde{\sigma}_{n+1}\left(\vec{r}^{\dot{x}_{1}} \ldots \vec{x}_{n} ; t\right) \sigma_{n+1}\left(\vec{r}^{\prime} \vec{x}_{1} \ldots \vec{x}_{n} ; t\right) .
\end{aligned}
$$

We note that since each of the ECCM amplitudes obeys the cluster property, the one-body density matrix exhibits the off-diagonal long-range order:

$$
\bar{\varrho}\left(\vec{r}, \vec{r}^{\prime} ; t\right) \underset{|\vec{r}-\vec{r}| \rightarrow \infty}{\longrightarrow} \tilde{\sigma}_{1}(\vec{r} ; t) \sigma_{1}\left(\vec{r}^{\prime} ; t\right),
$$

typical of a superfluid. We also see that it has the simple gauge-transformation property:

$$
\bar{\varrho}\left(\vec{r}, \vec{r}^{\prime} ; t\right) \rightarrow \bar{\varrho}^{\prime}\left(\vec{r}, \vec{r}^{\prime} ; t\right)=e^{i\left[A(\vec{r}, t)-\Lambda\left(\vec{r}^{\prime}, t\right]\right]} \underline{\varrho}\left(\vec{r}, \vec{r}^{\prime} ; t\right) .
$$

The local balance equations of hydrodynamics are now derived by transforming to relative and centre-of-mass coordinates:

$$
\vec{\xi}=\vec{r}-\vec{r}^{\prime} ; \quad \vec{R}=\frac{1}{2}\left(\vec{r}+\vec{r}^{\prime}\right),
$$

by expanding the equation of motion for $\bar{\varrho}\left(\vec{r}, \vec{r}^{\prime} ; t\right) \equiv \bar{\varrho}(\vec{R}: \vec{\xi} ; t)$ in powers of $\xi$, and by identifying at each order the pertinent gauge-invariant tensor by making use of Eqs. (84) and (89). Clearly, the zeroth-order equation is simply the number-density balance equation for $\bar{\varrho}(\vec{r}, t)$ itself. Similarly, the first- and secondorder equations in $\xi$ give respectively the balance equations for the (gauge-invariant) true current density $\vec{J}(\vec{r}, t)$ and the second-order kinetic stress tensor $K^{a b}(\vec{r}, t)$. In this way we build a hierarchy of exact local balance equations (for number conservation, momentum conservation, energy conservation, etc.). These are precisely the quantum-mechanical macroscopic laws of hydrodynamics for the system.

Furthermore, each of the macroscopic tensors involved (e.g., $\bar{\varrho}, \vec{J}, K^{a b}$ ) may be related back to its microscopic content in terms of the ECCM amplitudes $\left\{\sigma_{n}, \tilde{\sigma}_{n}\right\}$, as in Eq. (87). We further find, very importantly, that the hydrodynamical balance equations are also valid in such practical implementations as the SUB $(n)$ and other approximation schemes. Since all amplitudes $\left\{\sigma_{n}, \tilde{\sigma}_{n}\right\}$ obey the cluster property, the formalism is capable of properly handling all states of topological excitation or deformation, such as the vortex lines observed in liquid ${ }^{4} \mathrm{He}$. [100].

For further specific details, the interested reader is referred to the literature

\subsection{Charged impurity in a polarizable medium}

The technique of allowing low-energy positrons to annihilate inside metals, alloys, and other forms of condensed matter, has become an important experi- 
mental tool. Clearly, an $a b$ initio microscopic description of such a system comprising a positron embedded in an electron medium (or, more generally, a charged impurity particle inside a polarizable medium), should provide the most reliable interpretation of the experimental data. This system is clearly a prototype of the field-theoretical one-body problem (cf., the one-nucleon problem in the pion-nucleon field theory model of Sect. 7.2), and it is of particular interest to investigate it within the ECCM.

An obvious starting point is the ECCM description of the ket and bra states of the single impurity inside the electron system:

$$
\begin{array}{r}
\left|\Psi_{1}\right\rangle=\int d^{3} r b^{\dagger}(\vec{r}) \chi(\vec{r}, t) e^{S(\vec{r}, t)} e^{-\tilde{E}(\vec{r}, t)}|\Phi\rangle, \\
\left\langle\widetilde{\Psi}_{1}\right|=\int d^{3} r\langle\Phi| e^{\tilde{\Sigma}(\vec{r}, t)} e^{-S(\vec{r}, t)} \tilde{\chi}(\vec{r}, t) b(\vec{r}) .
\end{array}
$$

Here, the model state $|\Phi\rangle$ is an independent-particle (Slater determinant) model state for the electron system; $b(\vec{r})$ and $b^{\dagger}(\vec{r})$ are the destruction and creation operators (fields) for a positron at position $\vec{r}$; and the ECCM operators $S(\vec{r}, t)$ and $\widetilde{\Sigma}(\vec{r}, t)$ act in the electron Hilbert space to correlate the electrons among themselves as well as to describe the local enhancement of electrons around the positron. Thus, in the limit $r \rightarrow \infty, S$ and $\tilde{\Sigma}$ become the usual ECCM operators for the pure interacting electron gas. The functions $\chi$ and $\tilde{\chi}$ play the role of positron (ket and bra) wavefunctions, since the positron density may be written as:

$$
\varrho_{p}(\vec{r}, t)=\left\langle\tilde{\Psi}_{1}\left|b^{\dagger}(\vec{r}) b(\vec{r})\right| \Psi_{1}\right\rangle=\tilde{\chi}(\vec{r}, t) \chi(\vec{r}, t) .
$$

In coordinate-space representation, the ket wavefunction of Eq. (91), for example, becomes:

$$
\left\langle\vec{r} ; \vec{x}_{1} \ldots \vec{x}_{N} \mid \Psi_{1}\right\rangle=\chi(\vec{r}, t) \Psi_{\dot{r}}\left(\vec{x}_{1} \ldots \vec{x}_{N}\right),
$$

where $\vec{r}$ is the positron coordinate and $\left\{\vec{x}_{1} ; i=1,2, \ldots, N\right\}$ are the electron coordinates. It is very important to realize that the $N$-electron wavefunction $\Psi_{\vec{r}}\left(\vec{x}_{1} \ldots \vec{x}_{N}\right)$ depends crucially on the positron coordinate $\vec{r}$, due to the strong screening correlations and the enhancement of electron density around the positron. In the independent-particle model (where $S, \tilde{\Sigma} \rightarrow 0$ ), this $\vec{r}$-dependence is ignored, and the corresponding Slater determinant wavefunction typically underestimates the positron annihilation rate in metals, for example, by factors of the order of ten or more.

The ECCM now proceeds by applying a variational principle to the action functional:

$$
\mathscr{A}=\int d t \int d^{3} r\left\langle\tilde{\Psi}_{1}|(i \partial / \partial t-H)| \Psi_{1}\right\rangle
$$

It is shown in Ref. [101] that the ensuring discussion can be conveniently formulated in terms of an internal gauge field operator $\left\{A_{\mu}(\vec{r}, t) ; \mu=0,1,2,3\right\}$ :

$$
A_{\mu}=i e^{\Sigma} e^{-S} \frac{\partial}{\partial x_{\mu}} e^{S} e^{-\Sigma},
$$

where $x_{0}=i t, x_{1}=x, x_{2}=y, x_{3}=z$, which now describes the polarization of the electronic medium. In the usual fashion, the derivative operators $\left(\vec{\nabla}^{2}\right.$ and $\left.\partial / \partial t\right)$ in 
the Schrödinger equation may thereby be easily replaced by their covariant equivalents:

$$
\frac{\partial}{\partial x_{\mu}} \rightarrow D_{\mu}=\frac{\partial}{\partial x_{\mu}}-i A_{\mu},
$$

exactly as in electromagnetism, and also as in the previous example of Sect. 9.1. In this way, we can now derive equations of motion for the electronic ECCM coordinates $\left\{s_{n}, \tilde{\sigma}_{n}\right\}$ (or equivalently, $\left\{\sigma_{n}, \tilde{\sigma}_{n}\right\}$ ), as well as for the positron wavefunctions $\chi$ and $\tilde{\chi}$.

Just as in the previous example, we can also derive, for example, a continuity equation for the positron density, $\varrho_{p}$. One finds that from the viewpoint of the medium, the positron only enters into its microscopic equations of motion (for the amplitudes $\left.\left\{\sigma_{n}, \tilde{\sigma}_{n}\right\}\right)$ through the hydrodynamical positron density and current variables that describe its classical average flow. For further details the interested reader is referred to Ref. [101]. We note only that the general ECCM formalism provides an exact description of the positron motion in the polarizable medium. All of the usual conservation laws are satisfied, just as in Sect. 9.1. It may now be approximated by the usual $\operatorname{SUB}(n)$ scheme, for example.

\section{Concluding remarks}

One of our main aims in this survey has been to demonstrate the diversity, vitality, power, and accuracy of $\mathrm{CC}$ techniques, in the hope that some of the applications and some of the more formal developments that have been made in physics, might also prove useful within quantum chemistry. Such cross-fertilization is always beneficial. It may also be particularly useful now, when, for example, the systems of interest in ab initio quantum chemistry are becoming increasingly complex, and when extremely accurate wavefunctions are required for the currently proposed atomic physics tests for the violation of fundamental symmetries.

Of particular immediate interest with regard to the above comments, we mention only the potentially very powerful ECCM techniques on the one hand, and the very recent advent of several new approximation schemes for the otherwise exact $\mathrm{CC}$ equations on the other. We believe that it is worthwhile to explore both of these avenues further. In the first place, the ECCM is now rather well developed and understood at the formal level. In principle, as we have tried to convey, it has many advantages over both the CI method and the NCCM, particularly with regard to the more global aspects of a many-body system. For molecules, these include, for example, their overall topological structure and the similar global properties of their energy surfaces. Molecules which exhibit a "shape transition" between widely differing configurations when some internal (geometrical) parameter is varied beyond some critical value close to its equilibrium value, might therefore be prime initial candidates for study by the ECCM.

Although the generation of the actual ECCM equations is not difficult, we appreciate that their efficient computer-coding for practical implementations is no trivial task. Nevertheless, we believe that the possible rewards merit the effort. We even hazard the guess that within five to ten years the use of the ECCM in quantum chemistry will be accepted as a standard tool. Of course, for this prediction to be realized, the efficacy of sufficiently simple and robust truncation 
schemes will need to be demonstrated. Our optimism is based on the very wide corpus of successful applications that have already been made, and which have been reviewed here.

Secondly, we turn to the possible use of new truncation schemes other than the standard $\operatorname{SUB}(n)$ or $\operatorname{HCSUB}(n)$ schemes and their variants. We have already alluded to examples of these that have arisen in different contexts. One is based on the idea of trying to mimic within the CCM some of the successes of variational methods employing trial wavefunctions of the Jastrow type, for example. Thus, in the exact CCM equation for the matrix elements of the two-body partition $S_{2}$, say, of the cluster correlation operator, instead of setting the matrix elements of the higher-order partitions $S_{3}$ and $S_{4}$ to zero, as in the standard $\operatorname{SUB}(n)$ approximation, attempts have been made to approximate them in terms of $S_{2}$ itself. One way of doing this, loosely speaking, is to allocate them the values they would have if the exact many-body wavefunction were precisely of Jastrow type. This approach can also be systematically improved upon by enlarging the Jastrow trial wavefunctions to the more general Jastrow-Feenberg type of Eq. (4).

A second source of entirely new approximation schemes has been the application of the NCCM to spin-lattice problems. In this context, new approximations have been developed which concentrate on retaining configurations by criteria different from that used in the standard $\operatorname{SUB}(n)$ scheme, which focusses essentially on the maximum number of correlated "particles" (e.g., electron-hole pairs) defined with respect to the model state. One alternative scheme focussed instead on the number of "elementary excitations" (namely kinks in the quantum spin-lattice models considered). With an eye on the localized nature of the interaction and/or the excitations, another scheme focusses on the physical size or extent (in real space) of the "locale" over which the particles are correlated in the retained configurations. Both of these new physically motivated schemes may be precisely formulated in terms of systematic hierarchies, like that of the $\operatorname{SUB}(n)$ scheme. Preliminary investigations on discrete lattice models have been sufficiently encouraging to indicate that similarly motivated schemes might also be useful in quantum chemistry.

We conclude with some remarks on possible future developments and applications of $\mathrm{CC}$ techniques. For example, for extended systems, an important general area concerns inhomogeneous systems, for which we expect that the ECCM in particular should prove valuable. Indeed, the problem of a single charged impurity inside a polarizable medium discussed in Sect. 9.2 is a prototype here. Other important examples include surface phenomena and localization problems. In a sense, these problems are intermediate between such infinite, homogeneous many-body systems as the electron gas on the one hand, and such finite systems as atoms and molecules on the other. Since CC techniques have been successful in both limits, there are grounds to believe that they will continue to be so in this intermediate regime. In a similar vein, we also expect to see an increasing effort given to the use of $\mathrm{CC}$ techniques for the rigorous microscopic investigation of other many-body problems or field theories of current interest. These include such topical models of strongly-interacting electronic systems as the Hubbard model and its variants, and also such quantum field theories of fundamental interest as QCD, in both continuum and lattice-discretized versions.

We may also speculate on the use of $\mathrm{CC}$ techniques in quantum optics. A particular fundamental issue in this field concerns possible generalizations of 
the well-known Glauber coherent states of light of a single mode, associated with a bosonic creation operator $a^{\dagger}$. The (number-nonconserving) Glauber coherent states $|z\rangle$ are defined in terms of a unitary (and hence norm-preserving) operator, $U_{1}=U_{1}(z) \equiv \exp \left(z a^{\dagger}-z^{*} a\right)$, the displacement operator, acting on the vacuum state, $|0\rangle$. Similarly, the two-photon or "squeezed" coherent states, of much topical interest, are obtained via a unitary operator $U_{2}$, the squeezing operator, which is the exponential of an anti-Hermitian operator bilinear in the operators $a$ and $a^{\dagger}$, and which acts either on $|0\rangle$ or on $|z\rangle$. It is not difficult to show that the Glauber coherent states and the squeezed coherent states are, in the CCM language, just those states attainable from the cyclic vector $|0\rangle$ within the SUB(1) and SUB(2) subspaces respectively.

The problem with a naive generalization to "hypersqueezed" or $n$-photon coherent states with $n>2$, is that such $\mathrm{SUB}(n)$ states with $n>2$ are readily seen to have infinite norms. Hence, they are not realizable by simple finite unitary operators $U_{n}$, given as exponentials of anti-Hermitian operators which are themselves $n$ th-order multinomials in $a$ and $a^{\dagger}$. By contrast, within both the NCCM and ECCM we may define states $\left|\Psi_{n}\right\rangle$ and $\left\langle\widetilde{\Psi}_{n}\right|$ within the corresponding $\operatorname{SUB}(n)$ approximations, such that $\left\langle\Psi_{n} \mid \Psi_{n}\right\rangle=1$. As we have seen, the price paid is that $\left\langle\widetilde{\Psi}_{n}\right|$ is not then the Hermitian adjoint of $\left|\Psi_{n}\right\rangle$. Whether such $\operatorname{SUB}(n)$ hypersqueezed states will be of practical use in quantum optics, or whether they are in any sense physically realizable, remains to be seen. It does, however, seem to us that the theoretical mechanisms that have otherwise been proposed up till now to generate hypersqueezed states, are both much more convoluted and much less natural than the SUB $(n)$ states of either the NCCM or ECCM.

Turning to other needed developments of the CCM itself, we have already noted several instances of many-body systems with a degeneracy or near-degeneracy arising at a global rather than a local level. This typically arises where there are two or more degenerate configurations of the system, separated by a large potential barrier. The double-well quartic oscillator is an example. A similar molecular example is given by the double-degenerate, mirror-image, non-planar configurations of the ammonia $\left(\mathrm{NH}_{3}\right)$ molecule, the quantum tunneling between which leads to the closely-spaced states utilized in the ammonia maser. Corresponding periodic (infinite multi-well) generalizations lead to the band structure of energy levels in crystalline solids. The existing multireference versions of the CCM have grown from applications to finite open-shell systems, and are therefore much more attuned to the local degeneracy of a group of valence orbitals, for example. Their extension to situations of global degeneracy, typically associated with a breakdown of symmetry, is far from trivial. Nevertheless, its potential uses are varied and manifold.

Finally, we reiterate our belief that future advances in quantum many-body theory are likely to arise from bringing into conjunction the best elements of existing methods. We have already noted several examples of past and present activity in this area. We expect that quantum chemistry will play a full role in this regard, both in providing interesting arenas in which the confrontations and unions between methods can take place, and in suggesting new ingredients for incorporation into the CCM which have proven successful in other molecular applications. 


\section{References}

1. Mukherjee D (ed) (1989) Aspects of many-body effects in molecules and extended systems. (Lect Notes Chem, vol 50) Springer, Berlin Heidelberg New York

2. Bartlett RJ (1989) J Phys Chem 93:1697

3. Coester F (1958) Nucl Phys 7:421

4. Coester F, Kümmel H (1960) Nucl Phys 17:477

5. Čižek J (1966) J Chem Phys 45:4256; idem (1969) Advan Chem Phys 14:35

6. Paldus, J, Čižek J, Shavitt I (1972) Phys Rev A 5:50

7. Noga J, Bartlett RJ (1987) J Chem Phys 86:7041; idem (1988) ibid 89:3401(E)

8. Scuseria GE, Schaefer HF (1988) Chem Phys Lett 146:23; idem (1988) ibid 152:382

9. Bartlett RJ, Purvis GD (1978) Int J Quantum Chem 14:561; idem (1980) Phys Scr 21:251

10. Pople JA, Krishnan R, Schlegel HB, Binkley JS (1978) Int J Quantum Chem 14:545

11. Purvis GD, Bartlett RJ (1982) J Chem Phys 76:1910

12. Bartlett RJ (1981) Annu Rev Phys Chem 32:359

13. Bishop RF, Kümmel HG (1987) Phys Today 40(3):52

14. Binder K (ed) (1979) Monte Carlo methods in statistical physics. Springer, New York

15. Reynolds PJ, Ceperley DM, Alder BJ, Lester Jr. WA (1982) J Chem Phys 77:5593

16. Kalos MH, Whitlock PA (1986) Monte Carlo methods. Wiley, New York

17. Guardiola R (1988) Monte Carlo techniques in the many-body problem. In: Prosperi D, Rosati S, Violini G (eds) First international course on condensed matter. (ACIF Series, vol. 8) World Scientific, Singapore, $p 157$

18. Day BD (1978) Rev Mod Phys 50:495; idem (1979) Nucl Phys A328:1

19. Mahaux C (1979) Nucl Phys A328:24

20. Day BD (1981) in: Molinari A (ed) From nuclei to particles. North-Holland, Amsterdam

21. Bohm D, Pines D (1951) Phys Rev 82:625; idem (1953) ibid 92:609; Pines D (1953) ibid 92: 626

22. Gell-Mann M, Brueckner KA (1957) Phys Rev 106:364

23. Kümmel H, Lührmann KH, Zabolitzky JG (1978) Phys Rep 36C:1

24. Kümmel HG (1984) The coupled cluster method. In: Wu SS, Kuo TTS (eds) Nucleon-nucleon interaction and nuclear many-body problems. World Scientific, Singapore, p 46

25. Kvasnička V, Laurine V, Biskupič S (1979) Chem Phys Lett 73:81; Guest MF, Wilson S (1980) ibid 73:607; Frisch MJ, Krishnan R, Pople JA (1980) ibid 75:66; Bartlett RJ, Sekino H, Purvis GD (1983) ibid 98:66

26. Linderberg J, Öhrn Y (1973) Propagators in quantum chemistry. Academic, London

27. Öhrn Y (1989) Propagators for molecular electronic spectra. In: Mukherjee D (ed) Aspects of many-body effects in molecules and extended systems. (Lect Notes Chem, vol 50) Springer, Berlin Heidelberg New York, p 187; Oddershede J, Sengeløv PW (1989) Transition moments in RPA-like approximations. In: ibid, p 207

28. Diatlov IT, Sudakhov VV, Ter-Matirosian KA (1957) Sov Phys - JETP 5:631

29. Roulet R, Gavoret J, Nozières P (1969) Phys Rev 178:1072

30. Ripka G (1979) Phys Rep 56:1

31. Jackson AD, Lande A, Smith RA (1982) Phys Rep 86:55

32. Jackson AD, Lande A, Smith RA (1985) Phys Rev Lett 54:1469; Krotscheck E, Smith RA, Jackson AD (1986) Phys Rev A 33:3535

33. Lande A, Smith RA (1983) Phys Lett 131B:253

34. Smith RA, Jackson AD (1988) Fermion parquet: the approximations. In: Kallio AJ, Pajanne E, Bishop RF (eds) Recent progress in many-body theories, vol 1. Plenum, New York, p 327; Lande A, Smith RA (1988) Fermion parquet equations. In: ibid, p 335

35. Smith RA, Lande A (1988) Parquet theory: the diagrams. In: Arponen JS, Bishop RF, Manninen $M$ (eds) Condensed matter theories, vol 3. Plenum, New York, $p 1$

36. Jackson AD, Lande, A, Guitink RW, Smith RA (1985) Phys Rev B 31:403

37. Baym G, Kadanoff LP (1961) Phys Rev 124:287; Baym G (1962) ibid 127:1391

38. Smith RA (1989) Baym-Kadanoff theory made planar. In: Keller J (ed) Condensed matter theories, vol 4. Plenum, New York, p 129

39. Jastrow R (1955) Phys Rev 98:1479 
40. Iwamoto F, Yamada M (1957) Prog Theor Phys 17:543

41. Clark JW, Westhaus P (1968) J Math Phys 9:131; Westhaus P, Clark JW (1968) ibid 9:149

42. Gaudin M, Gillespie J, Ripka G (1971) Nucl Phys A176:237; Fantoni S, Rosati S (1974) Nuovo Cim 20A:179

43. Ripka G (1979) Nucl Phys A314:115

44. Feenberg E (1969) Theory of quantum fluids. Academic, New York

45. Fantoni S, Rosati S (1974) Lett Nuovo Cim 10:545; idem (1975) Nuovo Cim 25A:593

46. Krotscheck E, Ristig ML (1974) Phys Lett 48A:17; idem (1975) Nucl Phys A242:389

47. Clark JW (1979) in: Wilkinson DH (ed) Progress in particle and nuclear physics, vol 2. Pergamon, Oxford, p 89

48. Pandharipande VR, Wiringa RB (1979) Rev Mod Phys 51:821; Wiringa RB, Pandharipande VR (1979) Nucl Phys A317:1; Owen JC (1979) Phys Lett 82B:23; Lantto LJ, Siemens PJ (1979) Nucl Phys A317:55; Zabolitzky JG (1980) in: Negele JW, Vogt E (eds) Advances in nuclear physics, vol 12. Plenum, New York; Fantoni S, Pandharipande VR (1984) Nucl Phys A427:473

49. Rosati S, Fantoni S (1981) Correlations in infinite systems. In: Guardiola R, Ros J (eds) The many-body problem, Jastrow correlations versus Brueckner theory. (Lect Notes Phys, vol 138) Springer, Berlin Heidelberg New York, p 1

50. Rosati S, Viviani M (1988) in: Prosperi D, Rosati S, Violini G (eds) First international course on condensed matter. (ACIF Series, vol 8) World Scientific, Singapore, p 231

51. Ciofi degli Atti C (1986) Variational methods in the few-body problem. In: Bracci L et al (eds) Perspectives on theoretical nuclear physics. (Proc of the primo convegno su problemi di fisica nucleare teorica, Cortona, Italy, 1985) ETS editrice, Pisa, p 1

52. Clark JW (1979) Nucl Phys A328:587

53. Clark JW, Feenberg E (1959) Phys Rev 113:388; Jackson HW, Feenberg E (1961) Ann Phys (NY) 15:266; Feenberg E, Woo CW (1965) Phys Rev 137:A391; Clark JW, Westhaus P (1966) Phys Rev 141:833; idem (1966) ibid 149:990

54. Clark JW, Mead LR, Krotscheck E, Kürten KE, Ristig ML (1979) Nucl Phys A328:45

55. Krotscheck E, Clark JW (1979) Nucl Phys A328:73

56. Clark JW (1981) The correlated wave function approach to finite nuclear systems. In: Guardiola R, Ros J (eds) The many-body problem, Jastrow correlations versus Brueckner theory. (Lect Notes Phys, vol 138) Springer, Berlin Heidelberg New York, p 184

57. Chen JMC, Clark JW, Sandler DG (1982) Z Phys A305:223, 367

58. Krotscheck E, Smith RA, Clark JW, Panoff RM (1981) Phys Rev B 24:6383; Flynn MF, Clark JW, Krotscheck E, Smith RA, Panoff RM (1985) ibid 32:2945

59. Krotscheck E, Clark JW, Jackson AD (1983) Phys Rev B 28:5088

60. Clark JW, Krotscheck E, Schwesinger B (1984) Phys Lett 143B:287; idem (1985) Anales Fisica A81:116

61. Bishop RF (1988) Correlated basis functions and all that. In: Kallio AJ, Pajanne E, Bishop RF (eds) Recent progress in many-body theories, vol 1. Plenum, New York, p 385

62. Clark JW, Ristig ML (1973) Phys Rev C 7:1792; Ristig ML, Clark JW (1973) Nucl Phys A199:351

63. Mead LR, Clark JW (1980) Phys Lett 90B:331

64. Krotscheck E, Kümmel H, Zabolitzky JG (1980) Phys Rev A 22:1243; Krotscheck E, Clark JW (1981) Brueckner theory with Jastrow wave functions. In: Guardiola R, Ros J (eds) The many-body problem, Jastrow correlations versus Brueckner theory. (Lect Notes Phys, vol 138) Springer, Berlin Heidelberg New York, p 356

65. Nesbet RK (1958) Phys Rev 109:1632

66. Cauchy A (1821) Cours d'analyse de l'ecole polytechnique, ouvres complètes vols 2, 3; Macdonald JKL (1933) Phys Rev 43:830

67. Primas H (1965) in: Sinanoglu O (ed) Modern quantum chemistry, vol II. Academic, New York, $p 45$

68. Brueckner KA (1955) Phys Rev 97:1353; idem (1955) ibid 100:36

69. Lam PM, Clark JW, Ristig ML (1977) Phys Rev B 16:222; Clark JW, Lam PM, Zabolitzky JG, Ristig ML (1978) ibid 17:1147; Ristig ML, Kürten KE, Clark JW (1979) ibid 19:3539; Flynn MF, Clark JW, Panoff RM, Bohigas O, Stringari S (1984) Nucl Phys A427:253 
70. Chen JMC, Clark JW, Krotscheck E, Smith RA (1986) Nucl Phys A451:509

71. Clark JW, Krotscheck E (1984) in: Kümmel H, Ristig ML (eds) Recent progress in many-body theories. (Lect Notes Phys, vol 198) Springer, Berlin Heidelberg New York, p 127

72. Fantoni S, Wang X, Tosatti E, Lu Yu (1988) Physica C 153-155:1255; Wang XQ, Fantoni S, Tosatti E, Lu Yu (1990) Correlated spin-density-wave theory. In: Aguilera-Navarro VC (ed) Condensed matter theories, vol 5. Plenum, New York; Ristig ML (1990) Z Phys B 79: 351

73. Dabringhaus A, Ristig ML (1989) The $U(1)$ lattice gauge model: a correlated many-body system. In: Keller J (ed) Condensed matter theories, vol 4. Plenum, New York; idem (1991) The $U(1)_{3}$ lattice gauge vacuum. In: Fantoni S, Rosati S (eds) Condensed matter theories, vol 6. Plenum, New York

74. Arponen J (1983) Ann Phys (NY) 151:311

75. Bishop RF, Arponen J, Pajanne E (1989) Dynamic variational principles and extended coupled cluster techniques. In: Mukherjee D (ed) Aspects of many-body effects in molecules and extended systems. (Lect Notes Chem, vol 50) Springer, Berlin Heidelberg New York, p 79; Bishop RF, Arponen JS (1990) Int J Quantum Chem: Quantum Chem Symp 24:197

76. Bishop RF, Flynn MF, Boscá MC, Buendía E, Guardiola R (1990) J Phys G 16:L61; idem (1990) Exploring many-body theories in light nuclei. In: Greiner W, Stöcker H (eds) The nuclear equation of state, Part A: Discovery of nuclear shock waves and the EOS, Plenum, New York, p 605; idem (1990) Translationally invariant coupled cluster theory applied to the ${ }^{4} \mathrm{He}$ nucleus. In: Aguilera-Navarro VC (ed) Condensed matter theories, vol 5. Plenum, New York, p 255; Bishop RF, Buendia E, Flynn MF, Guardiola R (1990) Phys Rev C 42:1341

77. Bishop RF, Buendía E, Flynn MF, Guardiola R (1991) Variational cluster methods in coordinate space for small systems: center of mass corrections made easy. In: Fantoni S, Rosati $\mathrm{S}$ (eds) Condensed matter theories, vol 6. Plenum, New York

78. McGarry RG (1990) PhD thesis, University of Manchester; Bishop RF, Hughes SR, McGarry RG (1990) unpublished

79. Lipkin HJ, Meshkov N, Glick AJ (1965) Nucl Phys 62:188, 199, 211

80. Arponen JS, Bishop RF, Pajanne E (1987) Phys Rev A 36:2519; idem (1987) Extended coupled cluster method: quantum many-body theory made classical. In: Vashishta P, Kalia RK, Bishop RF (eds) Condensed matter theories, vol 2. Plenum, New York, p 357

81. (a) Emrich K (1981) Nucl Phys A351:379; (b) idem (1981) ibid A351:397; (c) Emrich K, Zabolitzky JG (1981) ibid A351:439

82. Bishop RF, Boscá MC, Flynn MF (1988) Phys Lett A132:440; idem (1989) Phys Rev A 40:3484

83. Bishop RF (1985) Anales Fisica A89:9; idem (1987) Towards a universal coupled cluster methodology for the various phases of condensed matter systems. In: Siemens PJ, Smith RA (eds) Recent progress in many-body theories. Texas A\&M University, College Station; Bishop RF, Piechocki W, Stevens GA (1988) Few-Body Systems 4:161, 179

84. Bishop RF (1984) Sum rules and a coupled cluster formulation of linear response theory. In: Kümmel H, Ristig ML (eds) Recent progress in many-body theories. (Lect Notes Phys, vol 198) Springer, Berlin Heidelberg New York, p 310; idem (1984) Linear response and sum rules in the coupled cluster formalism. In: Wu SS, Kuo TTS (eds) Nucleon-nucleon interaction and nuclear-many-body problems. World Scientific, Singapore, p 604

85. Bijl A (1940) Physica (Utrecht) 7:869; Feynman RP (1954) Phys Rev 94:262

86. Goldstone J (1957) Proc Roy Soc London A239:267

87. Brandow B (1967) Rev Mod Phys 39:771

88. Offermann R, Ey W, Kümmel H (1976) Nucl Phys A273:349; Offermann R (1976) ibid A273:368; Ey W (1978) ibid A296:189

89. Lindgren I (1978) Int J Quantum Chem: Quantum Chem Symp 12:33

90. Mukherjee D (1986) Chem Phys Lett 125:207; idem (1986) Int J Quantum Chem: Quantum Chem Symp 20:409

91. Lindgren I, Mukherjee D (1987) Phys Rep 151:93; Chowdhuri R, Mukherjee D, Prasad MD (1989) Separability problem in general many-electron systems. In: Mukherjee D (ed) Aspects of many-body effects in molecules and extended systems (Lect Notes Chem, vol 50). Springer, Berlin Heidelberg New York, p 3

92. Fink M (1974) Nucl Phys A221:163 
93. Hellmann H (1935) Acta Physicochimica USSR I(6):913; Feynman RP (1939) Phys Rev 56: 340

94. Thouless DJ (1961) The quantum mechanics of many-body systems. Academic, New York

95. Kümmel HG (1983) Int J Quantum Chem 24:79

96. Monkhorst HJ (1977) Int J Quantum Chem: Quantum Chem Symp 11:421

97. Bartlett RJ (1986) in: Jørgensen P, Simon J (eds) Geometrical derivatives of energy surfaces and molecular properties. Reidel, Dordrecht, p 35; Salter EA, Trucks GW, Bartlett RJ (1989) J Chem Phys 90:1752

98. Scheiner AC, Scuseria GE, Rice JE, Lee TJ, Schaefer HF (1987) J Chem Phys 87:5361

99. Arponen JS, Bishop RF, Pajanne E (1987) Phys Rev A 36:2539

100. Arponen J, Bishop RF, Pajanne E, Robinson NI (1988) Phys Rev A 37:1065; idem (1988) Towards a coupled cluster gauge-field approach to quantum hydrodynamics. In: Arponen JS, Bishop RF, Manninen M (eds) Condensed matter theories, vol 3. Plenum, New York, p 51; idem (1989) Quantum fluid dynamics: an extended coupled cluster treatment. In: Mukherjee D (ed) Aspects of many-body effects in molecules and extended systems (Lect Notes Chem, vol 50). Springer, Berlin Heidelberg New York, p 241

101. Arponen J, Bishop RF, Pajanne E (1987) On an effective gauge field description of a positron impurity in polarizable media. In: Vashishta P, Kalia RK, Bishop RF (eds) Condensed matter theories, vol 2. Plenum, New York, p 373

102. Robinson NI, Bishop RF, Arponen J (1989) Phys Rev A 40:4256; Bishop RF, Robinson NI, Arponen J (1990) Extended coupled cluster techniques for excited states: applications to quasispin models. In: Aguilera-Navarro VC (ed) Condensed matter theories, vol 5. Plenum, New York, p 329

103. (a) Arponen JS, Bishop RF (1990) Phys Rev Lett 64:111; (b) idem (1990) Coupled cluster parametrizations of model field theories and their Bargmann-space representations. In: Avishai Y (ed) Recent progress in many-body theories, vol 2. Plenum, New York; (c) Aalto E, Arponen JS, Bishop RF (1990) On the Bargmann space approach to the extended coupled cluster method for simple anharmonic systems. In: Aguilera-Navarro VC (ed) Condensed matter theories, vol 5. Plenum, New York, p 297; (d) Arponen JS, Bishop RF (1991) Ann Phys (NY) 207:171; (e) idem (1991) Theor Chim Acta 80

104. Arponen JS (1991) Theor Chim Acta 80

105. Altenbokum M, Emrich K, Kümmel H, Zabolitzky JG (1987) A temperature dependent coupled cluster method. In: Vashishta P, Kalia RK, Bishop RF (eds) Condensed matter theories, vol 2. Plenum, New York, p 389

106. Moszkowski SA (1988) Role of virtual double delta components in nuclei. In: Arponen JS, Bishop RF, Manninen M (eds) Condensed matter theories, vol 3. Plenum, New York, p 269

107. Zabolitzky JG, Ey W (1979) Nucl Phys A328:507

108. Day B (1981) Phys Rev Lett 47:226; Day B, Zabolitzky JG (1981) Nucl Phys A366:221

109. Kümmel HG (1979) Nucl Phys A317:199

110. (a) Bishop RF, Lührmann KH (1978) Phys Rev B 17:3757; (b) idem (1982) ibid 26:5523; (c) idem (1978) unpublished

111. Foldy LL (1961) Phys Rev 124:649; idem (1962) ibid 125:2208

112. Brueckner KA (1967) Phys Rev 156:204

113. Zabolitzky JG (1980) Phys Rev B 22:2353

114. Emrich K, Zabolitzky JG (1984) Phys Rev B 30:2049

115. Ceperley DM, Alder BJ (1980) Phys Rev Lett 45:566; Vosko SH, Wilk L, Nusair M (1980) Can J Phys 58:1200

116. Vashishta P, Singwi KS (1972) Phys Rev B 6:875, (E) 4883

117. Freeman DL (1977) Phys Rev B 15:5512; Arponen J, Pajanne E (1982) J Phys C 15:2665, 2683

118. Bishop RF, Lahoz WA (1987) J Phys A 20:4203; Lahoz WA, Bishop RF (1988) Z Phys B 73:363

119. (a) Lieb EH, Liniger W (1963) Phys Rev 130:1605; (b) idem (1963) ibid 130:1616

120. Agassi D, Lipkin HG, Meshkov N (1966) Nucl Phys 86:321

121. Lührmann KH (1977) Ann Phys (NY) 103:253

122. Arponen J (1982) J Phys G 8:L129; Arponen J, Rantakivi J (1983) Nucl Phys A407:141 
123. Giradeau M (1960) J Math Phys 1:516

124. Bethe HA (1931) Z Phys 71:205

125. Hughes SR (1990) PhD thesis, University of Manchester

126. Bender CM, Wu TT (1969) Phys Rev 184:1231; idem (1973) Phys Rev D 7:1620; Simon B (1970) Ann Phys (NY) 58:76

127. Hsue CS, Chern JL (1984) Phys Rev D 29:643

128. Kümmel H (1986) Preparing the ground for coupled cluster calculations. In: Malik FB (ed) Condensed matter theories, vol 1. Plenum, New York p 33

129. Kaulfuss UB, Altenbokum M (1986) Phys Rev D 33:3658

130. Kümmel HG (1988) The anharmonic oscillator revisited. In: Arponen JS, Bishop RF, Manninen $\mathbf{M}$ (eds) Condensed matter theories, vol 3. Plenum, New York, p 21

131. Bishop RF, Flynn MF (1988) Phys Rev A 38:2211

132. Kaulfuss U (1985) Phys Rev D 32:1421; Hsue CS, Kümmel H, Ueberholz P (1985) ibid 32:1435; Altenbokum M, Kümmel H (1985) ibid 32:2014; Kaulfuss U, Altenbokum M (1987) ibid 35:609; Funke M, Kaulfuss U, Kümmel H (1987) ibid 35:621

133. Kümmel H (1983) Phys Rev C 27:765; Hasberg G, Kümmel H (1986) ibid 33:1367

134. Anderson PW (1987) Science 235:1196

135. Roger M, Hetherington JH (1990) Phys Rev B 41:200

136. Bishop RF, Parkinson JB, Yang Xian (1991) Phys Rev B 43:13782; idem (1991) Theor Chim Acta 80:181

137. Haldane FDM (1983) Phys Lett A93:464; Affleck I, Haldane FDM (1987) Phys Rev B 36: 5291

138. Gross EP (1958) Ann Phys (NY) 4:57; idem (1961) Nuovo Cim 20:454; Pitaevskii LP (1961) Sov Phys - JETP 13:451; Fetter AL, Walecka JD (1971) Quantum theory of many-particle systems. McGraw-Hill, New York 